\title{
Quasi-periodic solutions of Schrödinger equations with quasi-periodic forcing in higher dimensional spaces
}

\author{
Min Zhang*, Jie Rui \\ College of Science, China University of Petroleum, Qingdao, Shandong 266580, People's Republic of China.
}

Communicated by K. Q. Lan

\begin{abstract}
In this paper, d-dimensional ( $\mathrm{dD}$ ) quasi-periodically forced nonlinear Schrödinger equation with a general nonlinearity

$$
i u_{t}-\Delta u+M_{\xi} u+\varepsilon \phi(t)\left(u+h\left(|u|^{2}\right) u\right)=0, \quad x \in \mathbb{T}^{d}, \quad t \in \mathbb{R}
$$

under periodic boundary conditions is studied, where $M_{\xi}$ is a real Fourier multiplier and $\varepsilon$ is a small positive parameter, $\phi(t)$ is a real analytic quasi-periodic function in $t$ with frequency vector $\omega=\left(\omega_{1}, \omega_{2} \ldots, \omega_{m}\right)$, and $h\left(|u|^{2}\right)$ is a real analytic function near $u=0$ with $h(0)=0$. It is shown that, under suitable hypothesis on $\phi(t)$, there are many quasi-periodic solutions for the above equation via KAM theory. (C)2017 All rights reserved.
\end{abstract}

Keywords: Quasi-periodically forced, KAM theory, Schrödinger equation, quasi-periodic solutions.

2010 MSC: 35Q41, 35G50.

\section{Introduction and main result}

Craig-Wayne-Bourgain method and infinite-dimensional KAM theory are two main approaches to deal with the problem of the existence of finite-dimensional tori for infinite-dimensional systems, such as Hamiltonian PDEs. The first one is a generalization of the Lyapunov-Schmidt reduction and the Newtonian method and allows one to avoid explicitly using the Hamiltonian structure of the systems. The reader is referred to Craig-Wayne [9], Bourgain [4-8]. The second approach is the extension of classical KAM theory. In this context the first results were obtained by Wayne [24], Kuksin [18], and Pöschel [21]. There are also many results on the existence of periodic solutions, quasi-periodic solutions for one dimensional Hamiltonian PDEs, see[1, 13, 14, 19-22, 25, 26] and the references therein. However, they meet difficulties in higher dimensional Hamiltonian PDEs.

Bourgain proved the existence of quasi-periodic solutions for partial differential equations in higher dimensional spaces with Dirichlet boundary conditions or periodic boundary conditions by the CraigWayne-Bourgain method. The reader is referred to Bourgain [6-8]. Berti-Bolle proved in [2, 3] that

\footnotetext{
*Corresponding author

Email addresses: zhangminmath@163.com (Min Zhang), rjhygl@163.com (Jie Rui)
} 
the wave equations and Schrödinger equations with a multiplicative potential on $\mathbb{T}^{\mathrm{d}}, \mathrm{d} \geqslant 1$, finitely differentiable nonlinearities admit the existence of quasi-periodic solutions via Nash-Moser method.

Geng-You [12, 15, 16] and Eliasson-Kuksin [11] constructed quasi-periodic solutions of higher dimensional Hamiltonian PDEs respectively by method from an infinite dimensional KAM theory. For the completely resonant cubic Schrödinger equation on a torus $\mathbb{T}^{\mathrm{d}}$, the existence of quasi-periodic solutions were proved by Procesi and Procesi [23]. Later Geng-You [17] proved an infinite dimensional KAM theory. They used the theorem to study the higher dimensional nonlinear Schrödinger equation

$$
i u_{t}-\Delta u+M \xi u+f\left(|u|^{2}\right) u=0, \quad x \in \mathbb{T}^{d}, \quad t \in \mathbb{R}
$$

with periodic boundary conditions.

All works mentioned above do not conclude the case with forced terms. It seems that the methods of the KAM for PDE theory have not been used to study the existence of quasi-periodic solutions for higher dimensional Schrödinger equations with quasi-periodic forcing of the form

$$
\mathrm{iu}_{\mathrm{t}}-\Delta \mathrm{u}+\mu \mathrm{u}+\mathrm{f}(\mathrm{t}, \mathrm{x}, \mathrm{u})=0, \quad \mu \geqslant 0
$$

with periodic boundary conditions.

In this paper, the existence and the linear stability of quasi-periodic solutions for quasi-periodically forced higher dimensional Schrödinger equation with periodic boundary conditions will be proved. This result has been obtained by Berti and Bolle [3]. However the approach used in the paper is based on KAM theory, while the proof in [3] is based on Lyapunov-Schmidt decomposition and a Nash-Moser scheme. Comparing with Nash-Moser method, the KAM approach has its own advantages what besides obtaining the existence of quasi-periodic solutions it allows one to construct a local normal form in a neighborhood of the obtained solutions, and provides more information of the dynamics, for instance on the stability of the solutions. More concretely, d-dimensional (dD) quasi-periodically forced nonlinear Schrödinger equation with a general nonlinearity

$$
i u_{t}-\Delta u+M \xi u+\varepsilon \phi(t)\left(u+h\left(|u|^{2}\right) u\right)=0, \quad x \in \mathbb{T}^{d}, \quad t \in \mathbb{R}
$$

under periodic boundary conditions

$$
u\left(t, x_{1}, x_{2}, \cdots, x_{d}\right)=u\left(t, x_{1}+2 \pi, x_{2}, \cdots, x_{d}\right)=\cdots=u\left(t, x_{1}, x_{2}, \cdots, x_{d-1}, x_{d}+2 \pi\right)
$$

is studied, where $M_{\xi}$ is a real Fourier multiplier and $\varepsilon$ is a small positive parameter, $\phi(t)$ is a real analytic quasi-periodic function in $t$ with frequency vector $\omega=\left(\omega_{1}, \omega_{2} \ldots, \omega_{m}\right) \subset[\tilde{\rho}, 2 \tilde{\rho}]^{m}$ for some constant $\tilde{\rho}>0$, and $h\left(|u|^{2}\right)$ is a real analytic function near $u=0$ with $h(0)=0$.

The main step is to reduce the equation (1.1) to a setting where KAM theory as developed by Geng-You [17] can be applied. This needs to reduce the linear part of Hamiltonian system to constant coefficients by a linear quasi-periodic change of variables with the same basic frequencies as the initial system. However, it cannot be guaranteed in general. A large part of the present paper will be devoted to the proof of reducibility of infinite-dimensional linear quasi-periodic systems. In fact, the question of reducibility of infinite-dimensional linear quasi-periodic systems is also interesting itself and remains open in the general case. There are two important works in this line. Firstly, Bambusi and Graffi [1] gave a general proof of reducibility of quasiperiodically forced PDEs. More recently, Kuksin and Eliasson [10] showed how such a problem can be reduced to a problem of persistence of tori, and thus solved by applying a standard KAM theorem. However, it would seem that their results cannot be directly applied to the problems in this paper because of the difference in the infinite dimensional KAM theories.

Assume that the operator $A=-\Delta+M_{\xi}$ with the periodic boundary conditions has eigenvalues $\lambda_{j}$ satisfying

$$
\lambda_{i_{l}}=\left|i_{l}\right|^{2}+\xi_{l}=i_{l 1}^{2}+\cdots+i_{l d}^{2}+\xi_{l}, \quad i_{l}=\left(i_{l 1}, \cdots, i_{l d}\right) \in \mathbb{Z}^{d}, \quad 1 \leqslant l \leqslant n,
$$




$$
\lambda_{j}=|j|^{2}=j_{1}^{2}+\cdots+j_{d}^{2}, \quad j=\left(j_{1}, \cdots, j_{d}\right) \in \mathbb{Z}^{d}, \quad j \neq i_{1}, \cdots, i_{n},
$$

and the corresponding eigenfunction $\phi_{j}(x)=\sqrt{\frac{1}{(2 \pi)^{d}}} e^{i<j, x>}$ form a basis in the domain of the operator. Denote $S=\left\{i_{1}=\left(i_{11}, \cdots, i_{1 d}\right), \cdots, i_{n}=\left(i_{n 1}, \cdots, i_{n d}\right)\right\} \subset \mathbb{Z}^{d}$ and assume that $i_{1}, \cdots, i_{n}$ are the distinguished sites of Fourier modes where $0 \in S$ in order to take care of $\left(\lambda_{j}, k\right)=(0,0)$, and the parameter $\xi=\left(\xi_{1}, \cdots, \xi_{n}\right) \in[0,1]^{n} \subset \mathbb{R}^{n}$.

In the present paper, the equation (1.1) is a small perturbation of the linear equation $i u_{t}=\Delta u-M_{\xi} u$ when $\varepsilon \ll 1$. The existence of the quasi-periodic solutions of equation (1.1) will be studied when $\varepsilon \neq 0$. For the purpose, throughout this paper, it will be assumed that

$(\mathrm{H}) \phi(t)$ is a real analytic quasi-periodic function in $t$ with frequency vector $\omega$, and $[\phi] \neq 0$ where $[\phi]$ denotes the time average of $\phi$, coinciding with the space average.

The following is the main result of this paper. The proof is based on a KAM theorem of PDE inspired by Geng-You [17].

Theorem 1.1 (Main theorem). Let $\tilde{\rho}$ be a positive constant, and $(\mathrm{H})$ holds. For given set $\mathrm{S}=\left\{i_{1}=\left(i_{11}, \cdots, i_{1 d}\right)\right.$, $\left.\cdots, i_{n}=\left(i_{n 1}, \cdots, i_{n d}\right)\right\} \subset \mathbb{Z}^{d}$ with $n \geqslant 2$, then for arbitrary $0<\gamma<1,0<\rho<1$ and $\gamma^{\prime}>0$ be small enough, there is small enough positive $\varepsilon^{*}\left(\rho, \gamma, \gamma^{\prime}\right)$ such that for any $0<\varepsilon<\varepsilon^{*}$, there exists a subset $\Omega \subset[\tilde{\rho}, 2 \tilde{\rho}]^{m}$ with meas $\Omega>(1-\gamma) \tilde{\rho}^{m}$ and there is a subset $\Sigma_{\gamma^{\prime}} \subset \Sigma:=\Omega \times[0,1]^{\mathrm{n}}$ with meas $\left(\Sigma \backslash \Sigma_{\gamma^{\prime}}\right)=\mathrm{O}\left(\gamma^{\prime}\right)$, such that for any $\left(\omega, \xi_{1}, \ldots, \xi_{n}\right) \in \Sigma_{\gamma^{\prime}}$, the nonlinear Schrödinger equation (1.1) possesses an $n$-dimensional quasi-periodic solution. Moreover, the obtained solutions are real analytic and linealy stable.

The rest of the paper is organized as follows. In Section 2 the Hamiltonian setting and reducibility of Schrödinger equations are discussed. In Section 3 the equations obtained in Section 2 are transformed into some partial Birkhoff normal forms for using the KAM theorem in [17]. In Section 4 it is introduced the infinite dimensional KAM theorem in [17] in order to prove the main result of Theorem 1.1. In Section 5 the main result of Theorem 1.1 is proved. Lemma 4.9 is proved in Appendix.

\section{Reducibility of Schrödinger equations}

Let us rewrite the Schrödinger equation (1.1) as follows

$$
i u_{t}=-A u-\varepsilon \phi(t)\left(u+h\left(|u|^{2}\right) u\right), \quad A=-\Delta+M_{\xi}, \quad x \in \mathbb{T}^{d}, \quad t \in \mathbb{R} .
$$

Equation (2.1) may be rewritten as

$$
u_{t}=i \frac{\partial H}{\partial \bar{u}}
$$

which may be viewed as the Hamiltonian system with Hamiltonian function

$$
\mathrm{H}=<A u, u>+\varepsilon \phi(t) \int_{\mathbb{T}^{d}}\left(|u|^{2}+\chi_{t}\left(|u|^{2}\right)\right) d x,
$$

where $\chi_{t}$ is a primitive of $h$ and $<\cdot, \cdot>$ denotes the scalar product in $L^{2}$.

We introduce coordinates $q=\left(\cdots, q_{j}, \cdots\right)_{j \in \mathbb{Z}^{d}}$ and its complex conjugate $\bar{q}=\left(\cdots, \bar{q}_{j}, \cdots\right)_{j \in \mathbb{Z}^{d}}$ through the relations

$$
u(t, x)=\sum_{j \in \mathbb{Z}^{d}} q_{j}(t) \phi_{j}(x)
$$

The coordinates are taken from some Hilbert space $l^{a}$ of sequences $q=\left(\cdots, q_{j}, \cdots\right)_{j \in \mathbb{Z}^{d}}$ with finite norm

$$
\|q\|_{a}=\sum_{j \in \mathbb{Z}^{\mathrm{d}}}\left|\mathrm{q}_{j}\right| e^{|j| a} .
$$


Below we will assume that $a \geqslant 0$. Let $\alpha \equiv\left(\ldots, \alpha_{j}, \ldots\right)_{j \in \mathbb{Z}^{d}}, \beta \equiv\left(\ldots, \beta_{j}, \ldots\right)_{j \in \mathbb{Z}^{d}}$, and $\alpha_{j}$ and $\beta_{j} \in \mathbb{N}$ with finitely many nonzero components of positive integers. The product $q^{\alpha} \bar{q}^{\beta}$ denotes $\prod_{j} q_{j}^{\alpha_{j}} \bar{q}_{j}^{\beta_{j}}$, $|\alpha|=\sum_{j \in \mathbb{Z}^{\mathrm{d}}} \alpha_{j},|\beta|=\sum_{j \in \mathbb{Z}^{\mathrm{d}}} \beta_{j}$. We obtain the Hamiltonian

$$
H=\Lambda+G
$$

where

$$
\Lambda=\sum_{j \in \mathbb{Z}^{\mathrm{d}}} \lambda_{j}\left|\mathrm{q}_{j}\right|^{2}+\varepsilon \phi(t)\left|\mathrm{q}_{j}\right|^{2}, \quad \mathrm{G} \equiv \varepsilon \phi(t) \int_{\mathbb{T}^{\mathrm{d}}} \chi_{\mathrm{t}}\left(|\mathrm{u}|^{2}\right) \mathrm{d} x=\varepsilon \phi(\mathrm{t}) \sum_{\alpha, \beta,|\alpha|+|\beta| \geqslant 4, \Sigma_{\mathbf{j} \in \mathbb{Z}^{\mathrm{d}}}\left(\alpha_{j}-\beta_{j}\right) j=0} \mathrm{G}_{\alpha \beta} \mathrm{q}^{\alpha} \overline{\mathrm{q}}^{\beta} .
$$

with $\left|G_{\alpha \beta}\right| \leqslant C, C>0$ is some absolute constant. The equations of motion are

$$
\dot{q}_{j}=i \frac{\partial H}{\partial \bar{q}_{j}}=i \lambda_{j} q_{j}+i \varepsilon \phi(t) q_{j}+i \frac{\partial G}{\partial \bar{q}_{j}}, \quad j \in \mathbb{Z}^{d}
$$

with respect to the symplectic structure $i \sum_{j \in \mathbb{Z}^{d}} d q_{j} \wedge d \bar{q}_{j}$ on $l^{a} \times l^{a}$.

Let $\varphi(\vartheta)$ be the shell of $\phi(t)$, we introduce a pair of action-angle variables $(J, \vartheta) \in \mathbb{R}^{\mathrm{m}} \times \mathbb{T}^{\mathrm{m}}$, then (2.2) can be written as a Hamiltonian system

$$
\dot{\vartheta}=\omega, \quad \dot{J}=-\frac{\partial H}{\partial \vartheta}, \quad \dot{q}_{j}=i \frac{\partial H}{\partial \bar{q}_{j}}, \quad j \in \mathbb{Z}^{\mathrm{d}}
$$

with the Hamiltonian

$$
H=<\omega, J>+\sum_{j \in \mathbb{Z}^{\mathrm{d}}}\left(\lambda_{j}+\varepsilon \varphi(\vartheta)\right)\left|\mathbf{q}_{j}\right|^{2}+\varepsilon \widehat{G},
$$

where

$$
\widehat{\mathrm{G}}=\sum_{\alpha, \beta,|\alpha|+|\beta| \geqslant 4, \sum_{j \in \mathbb{Z}^{d}}\left(\alpha_{j}-\beta_{j}\right) j=0} \widehat{\mathrm{G}}_{\alpha \beta}(\vartheta) q^{\alpha} \overline{\mathrm{q}}^{\beta}
$$

with

$$
\widehat{G}_{\alpha \beta}(\vartheta)= \begin{cases}\varphi(\vartheta) G_{\alpha \beta}, & \sum_{j \in \mathbb{Z}^{d}}\left(\alpha_{j}-\beta_{j}\right) j=0, \\ 0, & \sum_{j \in \mathbb{Z}^{d}}\left(\alpha_{j}-\beta_{j}\right) j \neq 0 .\end{cases}
$$

Denote

$$
\overline{\mathrm{H}}=<\omega, \mathrm{J}>+\sum_{j \in \mathbb{Z}^{\mathrm{d}}}\left(\lambda_{j}+\varepsilon \varphi(\vartheta)\right)\left|q_{j}\right|^{2},
$$

then

$$
\mathrm{H}=\overline{\mathrm{H}}+\varepsilon \widehat{\mathrm{G}} .
$$

In the following, we will investigate the reducibility of Hamiltonian (2.5). To make this reducibility, we introduce the following notations and spaces.

Define the sequences

$$
\sigma_{v}=\sigma_{0}\left(1-\frac{\sum_{s=1}^{v} s^{-2}}{2 \sum_{s=1}^{\infty} s^{-2}}\right), \quad v=1,2, \ldots
$$

and it is easy to see $\sigma_{v}>\sigma_{v+1}>\sigma_{0} / 2$. For some $\Gamma>0$ and a fixed $0<\rho<1$, we let

$$
\Gamma_{v}=\Gamma\left(1+C \sum_{s=v}^{+\infty} \varepsilon_{s}^{\rho}\right), \quad v=0,1, \ldots,
$$


where $\mathrm{C}$ is a constant that will be decided later. We let

$$
\begin{aligned}
\varepsilon_{0} & =\varepsilon, \quad \varepsilon_{v}=\varepsilon^{(1+\rho)^{v}}, \quad v=1,2, \ldots, \\
\Theta\left(\sigma_{v}\right) & =\left\{\vartheta=\left(\vartheta_{1}, \ldots, \vartheta_{m}\right) \in \mathbb{C}^{m} / 2 \pi \mathbb{Z}^{m}:\left|\operatorname{Im} \vartheta_{s}\right|<\sigma_{v}, s=1,2, \ldots, m\right\}, v=0,1,2, \ldots,
\end{aligned}
$$

and define

$$
\begin{aligned}
D_{v}^{a} & =\left\{(\vartheta, J, q, \bar{q}) \in \mathbb{C}^{m} / 2 \pi \mathbb{Z}^{m} \times \mathbb{C}^{m} \times l^{a} \times l^{a}:|\operatorname{Im} \vartheta|<\sigma_{v},|J|<\Gamma_{v}^{2},\|q\|_{a}<\Gamma_{v},\|\bar{q}\|_{a}<\Gamma_{v}\right\}, \\
v & =0,1,2, \ldots, \\
D_{\infty}^{a} & =\left\{(\vartheta, J, q, \bar{q}) \in \mathbb{C}^{m} / 2 \pi \mathbb{Z}^{m} \times \mathbb{C}^{m} \times l^{a} \times l^{a}:|\operatorname{Im} \vartheta|<\sigma_{0} / 2,|J|<\Gamma^{2}, \quad\|q\|_{a}<\Gamma,\|\bar{q}\|_{a}<\Gamma\right\},
\end{aligned}
$$

where $|\cdot|$ denotes the sup-norm for complex vectors and $l^{\mathrm{a}}$ denotes complex Hilbert space. For a one order Whitney smooth function $\mathrm{F}(\omega)$ on closed bounded set $\Omega^{*}$, we define

$$
\|\mathrm{F}\|_{\Omega^{*}}^{*}=\sup _{\omega \in \Omega^{*}}\left(|\mathrm{~F}|+\left|\partial_{\omega} \mathrm{F}\right|\right) .
$$

If $F(\omega)$ is a vector function from $\Omega^{*}$ to $l^{a, s}$ (or $\mathbb{R}^{m_{1} \times m_{2}}$ ) which is one order whitney smooth on $\Omega^{*}$, we define

$$
\|F\|_{a, \Omega^{*}}^{*}=\left\|\left(\left\|F_{i}(\omega)\right\|_{\Omega^{*}}^{*}\right)_{i}\right\|_{a} \quad\left(\text { or }\|F\|_{\Omega^{*}}^{*}=\max _{1 \leqslant i_{1} \leqslant m_{1}} \sum_{1 \leqslant i_{2} \leqslant m_{2}}\left(\left\|F_{i_{1} i_{2}}(\omega)\right\|_{\Omega^{*}}^{*}\right)\right) .
$$

For arbitrary $\sigma_{\mathrm{D}^{a}}>0, \Gamma_{\mathrm{D}^{a}}>0$, we let

$$
D^{a}=\left\{(\vartheta, J, q, \bar{q}) \in \mathbb{C}^{m} / 2 \pi \mathbb{Z}^{m} \times \mathbb{C}^{m} \times l^{a} \times l^{a}:|\operatorname{Im} \vartheta|<\sigma_{D^{a}},|J|<\Gamma_{D^{a}}^{2},\|q\|_{a}<\Gamma_{D^{a}},\|\bar{q}\|_{a}<\Gamma_{D^{a}}\right\} .
$$

Let $\tilde{w}=(\vartheta, J, q, \bar{q}) \in D^{a}$, we denote the weighted norm for $\tilde{w}$ by letting

$$
|\tilde{w}|_{a}=|\vartheta|+\frac{1}{\Gamma_{D^{a}}^{2}}|J|+\frac{1}{\Gamma_{D^{a}}}\|q\|_{a}+\frac{1}{\Gamma_{D^{a}}}\|\bar{q}\|_{a} .
$$

If $F(\eta ; \omega)$ is a vector function from $D^{a} \times \Omega^{*}$ to $l^{a}\left(\right.$ or $\mathbb{R}^{m_{1} \times m_{2}}$ ), which is one order whitney smooth on $\omega$, we define

$$
\|\mathrm{F}\|_{a, D^{a} \times \Omega^{*}}^{*}=\sup _{\eta \in D^{a}}\|F\|_{a, \Omega^{*}}^{*} \quad\left(\text { or }\|F\|_{D^{a} \times \Omega^{*}}^{*}=\sup _{\eta \in D^{a}}\|F\|_{\Omega^{*}}^{*}\right) \text {. }
$$

To function $F$, associate a Hamiltonian vector field defined as $X_{F}=\left\{F_{J},-F_{\vartheta}, i F_{\bar{q}},-i F_{q}\right\}$, we denote the weighted norm for $\mathrm{X}_{\mathrm{F}}$ by letting

$$
\left|X_{\mathrm{F}}\right|_{\mathrm{a}, \mathrm{D}^{a} \times \Omega^{*}}^{*}=\left\|\mathrm{F}_{\mathrm{J}}\right\|_{\mathrm{D}^{\mathrm{a}} \times \Omega^{*}}^{*}+\frac{1}{\Gamma_{\mathrm{D}^{\mathrm{a}}}^{2}}\left\|\mathrm{~F}_{\vartheta}\right\|_{\mathrm{D}^{\mathrm{a}} \times \Omega^{*}}^{*}+\frac{1}{\Gamma_{\mathrm{D}^{\mathrm{a}}}}\left\|\mathrm{F}_{\bar{z}}\right\|_{\mathrm{a}, \mathrm{D}^{\mathrm{a}} \times \Omega^{*}}^{*}+\frac{1}{\Gamma_{\mathrm{D}^{\mathrm{a}}}}\left\|\mathrm{F}_{\mathrm{q}}\right\|_{\mathrm{a}, \mathrm{D}^{\mathrm{a}} \times \Omega^{*}}^{*}
$$

Let $w=(\mathrm{q}, \overline{\mathrm{q}}) \in \mathrm{l}^{\mathrm{a}} \times \mathrm{l}^{\mathrm{a}}$ be a doubly infinite complex sequence. Let $\mathrm{A}(\eta ; \omega)$ be an operator from $l^{\mathrm{a}} \times l^{\mathrm{a}}$ to $l^{a} \times l^{a}$ for $(\eta ; \omega) \in D^{a} \times \Omega^{*}$, we define the norm

$$
\begin{aligned}
\|w\|_{a} & =\|q\|_{a}+\|\bar{q}\|_{a}, \\
\|A(\eta ; \omega)\|_{a, D^{a} \times \Omega^{*}} & =\sup _{(\eta ; \omega) \in D^{a} \times \Omega^{*}} \sup _{w \neq 0} \frac{\|A(\eta ; \omega) w\|_{a}}{\|w\|_{a}}, \\
\|A(\eta ; \omega)\|_{a, D^{a} \times \Omega^{*}}^{*} & =\|A\|_{a, D^{a} \times \Omega^{*}}+\left\|\partial_{\omega} A\right\|_{a, D^{a} \times \Omega^{*}}
\end{aligned}
$$

Let $B(\eta ; \omega)$ be an operator from $D^{a}$ to $D^{a}$ for $(\eta ; \omega) \in D^{a} \times \Omega^{*}$, we define the operator norm

$$
|\mathrm{B}(\eta ; \omega)|_{\mathrm{a}, \mathrm{D}^{\mathrm{a}} \times \Omega^{*}}=\sup _{(\eta ; \omega) \in \mathrm{D}^{\mathrm{a}} \times \Omega^{*} \tilde{w} \neq 0} \frac{|\mathrm{B}(\eta ; \omega) \tilde{w}|_{\mathrm{a}}}{|\tilde{w}|_{\mathrm{a}}}, \quad|\mathrm{B}(\eta ; \omega)|_{\mathrm{a}, \mathrm{D}^{\mathrm{a}} \times \Omega^{*}}^{*}=|\mathrm{B}|_{\mathrm{a}, \mathrm{D}^{\mathrm{a}} \times \Omega^{*}}+\left|\partial_{\omega} \mathrm{B}\right|_{\mathrm{a}, \mathrm{D}^{\mathrm{a}} \times \Omega^{*}} .
$$


Let $0 \neq k \in \mathbb{Z}^{\mathrm{m}}$, and let

$$
\mathcal{R}_{\mathrm{k}}^{1}=\left\{\omega \in[\tilde{\rho}, 2 \tilde{\rho}]^{m}:|<k, \omega>| \leqslant \frac{\tilde{\rho}}{C_{*}|k|^{m+1}}\right\},
$$

we have

$$
\operatorname{meas} \mathcal{R}_{\mathrm{k}}^{1} \leqslant \mathrm{C}_{1}|\mathrm{k}|^{-1} \tilde{\rho}^{\mathrm{m}-1} \frac{\tilde{\rho}}{\mathrm{C}_{*}|\mathrm{k}|^{\mathrm{m}+1}} \leqslant \frac{\mathrm{C}_{1}}{\mathrm{C}_{*}|\mathrm{k}|^{\mathrm{m}+2}} \tilde{\rho}^{\mathrm{m}} .
$$

Let $\Omega^{1}=\bigcup_{0 \neq k \in \mathbb{Z}^{m}} \mathcal{R}_{k^{\prime}}^{1}$, we get

$$
\text { meas } \Omega^{1} \leqslant \sum_{0 \neq k \in \mathbb{Z}^{m}} \operatorname{meas} \mathcal{R}_{k}^{1} \leqslant C_{1} C_{*}^{-1} \tilde{\rho}^{m} \sum_{0 \neq k \in \mathbb{Z}^{m}} \frac{1}{|k|^{m+2}} \leqslant C_{2} C_{*}^{-1} \tilde{\rho}^{m} .
$$

For arbitrary fixed $0<\gamma<1$, we have

$$
\operatorname{meas} \Omega^{1} \leqslant \gamma \tilde{\rho}^{m}
$$

if $C_{*} \gg 1$. Let $\Omega=[\tilde{\rho}, 2 \tilde{\rho}]^{m} \backslash \Omega^{1}$, we get

$$
\text { meas } \Omega \geqslant(1-\gamma) \tilde{\rho}^{m} \text {. }
$$

Lemma 2.1. For given $\sigma_{0}>0$, and arbitrary fixed $0<\gamma<1,0<\rho<1$ there is an $\varepsilon^{*}(\gamma)>0$ such that for any $0<\varepsilon<\varepsilon^{*}(\gamma)$ and $\omega \in \Omega$, there is a real analytic canonical transformation $\Sigma_{\infty}^{0}$ defined on $\mathrm{D}_{\infty}^{\mathrm{a}} \times \Omega$ such that the following statements hold:

(i) there is some absolute constant $\mathrm{C}>0$ independent on $\mathrm{j}$ such that

$$
\left|\Sigma_{\infty}^{0}-i d\right|_{a, D_{\infty}^{a} \times \Omega}^{*} \leqslant \mathcal{C}^{\rho},
$$

where id is the identity mapping;

(ii) the transformation $\Sigma_{\infty}^{0}$ changes Hamiltonian (2.5) into

$$
\overline{\mathrm{H}} \circ \Sigma_{\infty}^{0}=<\omega, \mathrm{J}>+\sum_{j \in \mathbb{Z}^{\mathrm{d}}} \mu_{j}\left|q_{j}\right|^{2},
$$

where

$$
\mu_{j}=\lambda_{j}+\varepsilon[\phi]+\varepsilon^{(1+\rho)} \mu_{j}^{*}, \quad\left\|\mu_{j}^{*}\right\|_{\Omega}^{*} \leqslant C, \quad j \in \mathbb{Z}^{d},
$$

and for any fixed $\mathfrak{j} \in \mathbb{Z}^{\mathrm{d}}, \tilde{\mathfrak{j}} \in \mathbb{Z}^{\mathrm{d}} \backslash\{0\}$, the limits $\lim _{\tilde{\mathfrak{t}} \rightarrow \infty} \mu_{\mathfrak{j}+\tilde{\mathfrak{j}} \tilde{\mathfrak{t}}}^{*}$ and $\lim _{\tilde{\mathfrak{t}} \rightarrow \infty} \partial_{\omega} \mu_{\mathfrak{j}+\tilde{\mathfrak{j}} \tilde{\mathfrak{t}}}^{*}$ exist and

$$
\left\|\mu_{j+\tilde{j} \tilde{\mathfrak{t}}}^{*}-\lim _{\tilde{\mathfrak{t}} \rightarrow \infty} \mu_{j+\tilde{j} \tilde{\mathfrak{t}}}^{*}\right\|_{\Omega}^{*} \leqslant \frac{1}{|\tilde{\mathfrak{t}}|} .
$$

Proof. First of all, we will construct iteratively a series $\left\{\mathrm{H}_{l}\right\}$ of Hamiltonian functions of the form

$$
\mathrm{H}_{\mathrm{l}}=\mathrm{H}_{\mathrm{l}}^{2}+\mathrm{R}_{\mathrm{l}}, \quad \mathrm{l}=0,1, \ldots, \mathrm{v},
$$

where

$$
H_{l}^{2}:=<\omega, J>+\sum_{j \in \mathbb{Z}^{\mathrm{d}}} \lambda_{j, l} q_{j} \bar{q}_{j}, \quad R_{l}:=\varepsilon_{l} \sum_{j \in \mathbb{Z}^{\mathrm{d}}} R_{j, l}(\vartheta, \omega) q_{j} \bar{q}_{j},
$$

with $R_{j, l}(\vartheta, \omega)=\sum_{k \in \mathbb{Z}^{m}} R_{j, l, k}(\omega) e^{i<k, \vartheta>}$ and $R_{j, 0}=\varphi(\vartheta)$. Furthermore

$$
\left\|R_{j, l}\right\|_{\Theta\left(\sigma_{l}\right) \times \Omega}^{*} \leqslant C, \quad l=0,1, \ldots, v,
$$

and for any fixed $j \in \mathbb{Z}^{\mathrm{d}}, \tilde{\mathfrak{j}} \in \mathbb{Z}^{\mathrm{d}} \backslash\{0\}$, the limits $\lim _{\tilde{\mathfrak{t}} \rightarrow \infty} \mathrm{R}_{\mathbf{j}+\tilde{j} \tilde{\mathrm{t}}, l}$ and $\lim _{\tilde{\mathfrak{t}} \rightarrow \infty} \partial_{\omega} \mathrm{R}_{\mathbf{j}+\tilde{j} \tilde{\mathfrak{t}}, l}$ exist and

$$
\left\|R_{\mathfrak{j}+\tilde{j} \tilde{\mathbf{t}}, l}-\lim _{\tilde{\mathfrak{t}} \rightarrow \infty} R_{\mathbf{j}+\tilde{\mathbf{j}} \tilde{t}, l}\right\|_{\Theta\left(\sigma_{\mathfrak{l}}\right) \times \Omega}^{*} \leqslant \frac{1}{|\tilde{\mathfrak{t}}|} .
$$


And

$$
\lambda_{j, 0}=\lambda_{j}, \quad \lambda_{j, l}=\lambda_{j}+\sum_{s=0}^{l-1} \mu_{j, l, s}, \quad l \geqslant 1,
$$

here

$$
\mu_{j, l, 0}=\varepsilon[\phi], \quad \mu_{j, l, s}=\varepsilon_{s} \mu_{j, l, s}^{*}, \quad\left\|\mu_{j, l, s}^{*}\right\|_{\Omega}^{*} \leqslant C, \quad s=1,2, \ldots, v,
$$

and for any fixed $\mathfrak{j} \in \mathbb{Z}^{\mathrm{d}}, \tilde{j} \in \mathbb{Z}^{\mathrm{d}} \backslash\{0\}$, the limits $\lim _{\tilde{\mathfrak{t}} \rightarrow \infty} \mu_{\mathfrak{j}+\tilde{\mathfrak{j}} \tilde{\mathrm{t}}, l, s}^{*}$ and $\lim _{\tilde{\mathfrak{t}} \rightarrow \infty} \partial_{\omega} \mu_{\mathfrak{j}+\tilde{\mathfrak{j}}, \mathrm{l}, \mathrm{s}}^{*}$ exist and

$$
\left\|\mu_{j+\tilde{j} \tilde{\mathfrak{t}}, l, s}^{*}-\lim _{\tilde{\mathfrak{t}} \rightarrow \infty} \mu_{\mathfrak{j}+\tilde{\mathrm{j}} \tilde{\mathrm{t}}, \mathrm{l}, \mathrm{s}}^{*}\right\|_{\Omega}^{*} \leqslant \frac{1}{|\tilde{\mathfrak{t}}|} .
$$

Clearly, we have that $H_{0}=\bar{H}$ for $l=0$, and $\left\|R_{j, 0}\right\|_{\Theta\left(\sigma_{0}\right) \times \Omega}^{*} \leqslant C$, where $C$ is an absolute constant

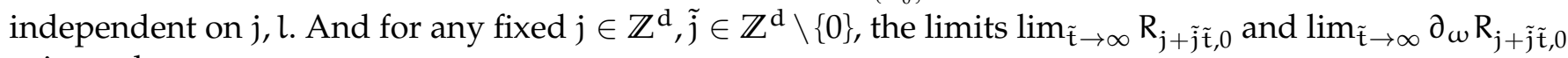
exist and

$$
\left\|R_{j+\tilde{j} \tilde{t}, 0}-\lim _{\tilde{\mathfrak{t}} \rightarrow \infty} R_{\mathbf{j}+\tilde{\mathfrak{j}} \tilde{\mathrm{t}}, 0}\right\|_{\Theta\left(\sigma_{l}\right) \times \Omega}^{*}=0 \leqslant \frac{1}{|\tilde{\mathfrak{t}}|} .
$$

Let $X_{\mathcal{F}_{v}}$ be the Hamiltonian vector field associated with a function $\mathcal{F}_{v}$ :

$$
\mathcal{F}_{v}=\varepsilon_{v} F_{v}=\varepsilon_{v} \sum_{j \in \mathbb{Z}^{\mathrm{d}}} f_{j, v}(\vartheta ; \omega) q_{j} \bar{q}_{j}
$$

with

$$
f_{j, v}(\vartheta ; \omega)=\sum_{k \in \mathbb{Z}^{m}} f_{j, v, k}(\omega) e^{i<k, \vartheta>}
$$

and $\left[f_{j, v}\right]=0$, and let $X_{\mathcal{F}_{v}}^{t}$ denote its time-t map.

We look for a change of variables $S_{v}$ defined on a domain $D_{v+1}^{a}$ by the time-one map $X_{\mathcal{F}_{v}}^{1}$ of the Hamiltonian vector field $X_{\mathcal{F}_{v}}$, such that the system $(2.7)_{v}$ is transformed into the form $(2.7)_{v+1}$ and satisfies $(2.8)_{v+1},(2.9)_{v+1},(2.10)_{v+1},(2.11)_{v+1}$, and (2.12) $)_{v+1}$. In fact, the new Hamiltonian $\mathrm{H}_{v+1}$ can be written as

$$
\begin{aligned}
\mathrm{H}_{v+1} & :=\mathrm{H}_{v} \circ X_{\mathcal{F}_{v}}^{1} \\
& =\mathrm{H}_{v}^{2}+\mathrm{R}_{v}+\left\{\mathrm{H}_{v}^{2}, \mathcal{F}_{v}\right\}+\varepsilon_{v} \int_{0}^{1}(1-t)\left\{\left\{\mathrm{H}_{v}^{2}, \mathcal{F}_{v}\right\}, \mathrm{F}_{v}\right\} \circ X_{\mathcal{F}_{v}}^{\mathrm{t}} \mathrm{dt}+\varepsilon_{v} \int_{0}^{1}\left\{\mathrm{R}_{v}, \mathrm{~F}_{v}\right\} \circ X_{\mathcal{F}_{v}}^{\mathrm{t}} \mathrm{dt} .
\end{aligned}
$$

The function $F_{v}$ is determined by the homological equation

$$
\mathrm{R}_{v}+\left\{\mathrm{H}_{v}^{2}, \mathcal{F}_{v}\right\}=\varepsilon_{v} \sum_{j \in \mathbb{Z}^{\mathrm{d}}}\left[\mathrm{R}_{j, v}\right] \mathrm{q}_{j} \overline{\mathrm{q}}_{j},
$$

which is equivalent to

$$
-<\omega, \partial_{\vartheta} f_{j, v}(\vartheta ; \omega)>+R_{j, v}(\vartheta ; \omega)=\left[R_{j, v}\right] .
$$

Inserting (2.13) into (2.15) we get

$$
i<k, \omega>f_{j, v, k}(\omega)=R_{j, v, k}(\omega), \quad k \neq 0 .
$$

Hence, we get

$$
f_{j, v}(\vartheta ; \omega)=\sum_{0 \neq k \in \mathbb{Z}^{m}} \frac{R_{j, v, k}(\omega)}{i<k, \omega>} e^{i<k, \vartheta>} .
$$

We can get by Cauchy's estimate and $(2.8)_{v}$,

$$
\left|R_{j, v, k}\right| \leqslant\left\|R_{j, v}\right\|_{\Theta\left(\sigma_{v}\right) \times \Omega}^{*} e^{-|k| \sigma_{v}} \leqslant C e^{-|k| \sigma_{v}}
$$


and

$$
\left|\partial_{\omega} R_{j, v, k}\right| \leqslant\left\|R_{j, v}\right\|_{\Theta\left(\sigma_{v}\right) \times \Omega}^{*} e^{-|k| \sigma_{v}} \leqslant C e^{-|k| \sigma_{\nu}} .
$$

By Cauchy's estimate and (2.9) $)_{v}$, we have for any fixed $j \in \mathbb{Z}^{\mathrm{d}}, \tilde{j} \in \mathbb{Z}^{\mathrm{d}} \backslash\{0\}$, the limits $\lim _{\tilde{\mathfrak{t}} \rightarrow \infty} R_{\mathfrak{j}+\tilde{j} \tilde{\mathrm{t}}, v, k}$ and $\lim _{\tilde{\mathfrak{t}} \rightarrow \infty} \partial_{\omega} R_{\mathfrak{j}+\tilde{j} \tilde{\mathfrak{t}}, v, k}$ exist and

$$
\left\|R_{\mathbf{j}+\tilde{j} \tilde{t}, v, k}-\lim _{\tilde{\mathfrak{t}} \rightarrow \infty} R_{\mathbf{j}+\tilde{j} \tilde{\mathbf{t}}, v, k}\right\|_{\Omega}^{*} \leqslant \frac{1}{|\tilde{t}|} .
$$

Thus, we have

$$
\sup _{(\vartheta ; \omega) \in \Theta\left(\sigma_{v+1}\right) \times \Omega}\left|f_{j, v}\right| \leqslant C C_{*} \tilde{\rho}^{-1} \sum_{0 \neq k \in \mathbb{Z}^{m}}|k|^{m+1} e^{-\sigma_{v}|k|} e^{\sigma_{v+1}|k|} .
$$

So, using Lemma 3.3 in [26], we get, for $(\vartheta ; \omega) \in \Theta\left(\sigma_{v+1}\right) \times \Omega$,

$$
\left|f_{j, v}\right| \leqslant C C_{*} \tilde{\rho}^{-1}(v+1)^{4 m+2} \leqslant C(v+1)^{6 m+6},
$$

where $C:=C C_{*} \tilde{\rho}^{-1}$. Moreover, by $(2.10)_{v}$ and $(2.11)_{v}$, it follows that

$$
\left|\partial_{\omega} \lambda_{j, v}\right| \leqslant C \varepsilon
$$

Thus, in view of (2.16)-(2.18) and (2.21), we have, for $(\vartheta ; \omega) \in \Theta\left(\sigma_{v+1}\right) \times \Omega$,

$$
\begin{aligned}
\left|\partial_{\omega} f_{j, v}\right| & \leqslant \sum_{0 \neq k \in \mathbb{Z}^{m}}\left(\left|\frac{\partial_{\omega} R_{j, v, k}(\omega)}{<k, \omega>}\right|+\left|\frac{R_{j, v, k}(\omega) k}{<k, \omega>^{2}}\right|\right)\left|e^{i<k, \vartheta>}\right| \\
& \leqslant C \sum_{0 \neq k \in \mathbb{Z}^{m}}\left(C_{*} \tilde{\rho}^{-1}|k|^{m+1}+|k|\left(C_{*} \tilde{\rho}^{-1}|k|^{m+1}\right)^{2}\right) e^{-\sigma_{v}|k|} e^{\sigma_{v+1}|k|} \\
& \leqslant 2 C C_{*}^{2} \tilde{\rho}^{-2} \sum_{0 \neq k \in \mathbb{Z}^{m}}|k|^{2 m+3} e^{-\sigma_{v}|k|} e^{\sigma_{v+1}|k|} \\
& \leqslant 2 C C_{*}^{2} \tilde{\rho}^{-2}(v+1)^{6 m+6} \leqslant C(v+1)^{6 m+6}
\end{aligned}
$$

where $C:=2 C \cdot C_{*}^{2} \rho^{-2}$. In view of (2.20) and (2.22) we have

$$
\left\|f_{j, v}\right\|_{\Theta\left(\sigma_{v+1}\right) \times \Omega}^{*} \leqslant C(v+1)^{6 m+6},
$$

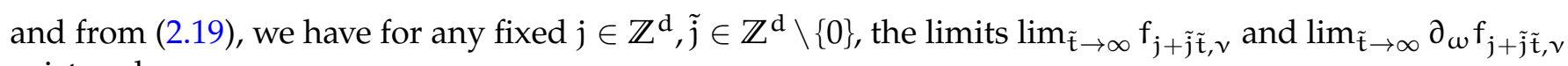
exist and

$$
\left\|f_{j+\tilde{j} \tilde{\mathfrak{t}}, v}-\lim _{\tilde{\mathfrak{t}} \rightarrow \infty} f_{j+\tilde{j} \tilde{t}, v}\right\|_{\Theta\left(\sigma_{v+1}\right) \times \Omega}^{*} \leqslant \frac{1}{|\tilde{t}|}(v+1)^{6 m+6} .
$$

In view of (2.16), we have

$$
\partial_{\vartheta} f_{j, v}(\vartheta ; \omega)=\sum_{0 \neq k \in \mathbb{Z}^{m}} \frac{R_{j, v, k}(\omega)}{<k, \omega>} e^{i<k, \vartheta>} \cdot k, \quad \partial_{\vartheta \vartheta} f_{j, v}(\vartheta ; \omega)=\sum_{0 \neq k \in \mathbb{Z}^{m}} \frac{R_{j, v, k}(\omega)}{<k, \omega>} e^{i<k, \vartheta>} \cdot i k k^{\top},
$$

where $k$ is an $m$ column vector and $k k^{\top}$ is an $m \times m$ matrix. Similar to above discussion, we get the following estimates

$$
\begin{array}{r}
\left\|\partial_{\vartheta} f_{j, v}\right\|_{\Theta\left(\sigma_{v+1}\right) \times \Omega}^{*} \leqslant C(v+1)^{6 m+8}, \\
\left\|\partial_{\vartheta \vartheta} f_{j, v}\right\|_{\Theta\left(\sigma_{v+1}\right) \times \Omega}^{*} \leqslant C(v+1)^{6 m+10}
\end{array}
$$


for any fixed $j \in \mathbb{Z}^{\mathrm{d}}, \tilde{j} \in \mathbb{Z}^{\mathrm{d}} \backslash\{0\}$, the limits $\lim _{\tilde{\mathfrak{t}} \rightarrow \infty} \partial_{\vartheta} f_{\mathfrak{j}+\tilde{\mathfrak{j}}, v}, \lim _{\tilde{\mathfrak{t}} \rightarrow \infty} \partial_{\omega} \partial_{\vartheta} f_{\mathfrak{j}+\tilde{j} \tilde{\mathfrak{t}}, v}, \lim _{\tilde{\mathfrak{t}} \rightarrow \infty} \partial_{\vartheta \vartheta} f_{j+\tilde{j} \tilde{\mathfrak{t}}, v}$, and $\lim _{\tilde{\mathfrak{t}} \rightarrow \infty} \partial_{\omega} \partial_{\vartheta \vartheta} f_{j+\tilde{j} \tilde{\mathfrak{t}}, v}$ exist and

$$
\begin{array}{r}
\left\|\partial_{\vartheta} f_{j+\tilde{j} \tilde{\mathfrak{t}}, v}-\lim _{\tilde{\mathfrak{t}} \rightarrow \infty} \partial_{\vartheta} f_{j+\tilde{j} \tilde{\mathbf{t}}, v}\right\|_{\Theta\left(\sigma_{v+1}\right) \times \Omega}^{*} \leqslant \frac{1}{|\tilde{\mathfrak{t}}|}(v+1)^{6 m+8}, \\
\left\|\partial_{\vartheta \vartheta} f_{j+\tilde{j} \tilde{\mathfrak{j}}, v}-\lim _{\tilde{\mathfrak{t}} \rightarrow \infty} \partial_{\vartheta \vartheta} f_{j+\tilde{j} \tilde{\mathfrak{j}}, v}\right\|_{\Theta\left(\sigma_{v+1}\right) \times \Omega}^{*} \leqslant \frac{1}{|\tilde{\mathfrak{t}}|}(v+1)^{6 m+10} .
\end{array}
$$

Let

$$
\lambda_{j, v+1}=\lambda_{j, v}+\varepsilon_{v}\left[R_{j, v}\right],
$$

then by $(2.8)_{v}$ and $(2.9)_{v}$ it is easy to see that $\lambda_{j, v+1}$ satisfies the conditions $(2.10)_{v+1},(2.11)_{v+1}$, and $(2.12)_{v+1}$.

To get the estimates for the flow $X_{\mathcal{F}}^{\mathrm{t}}$, we let

$$
B_{j, v}(\vartheta ; \omega)=\left(\begin{array}{cc}
0 & f_{j, v}(\vartheta ; \omega) \\
f_{j, v}(\vartheta ; \omega) & 0
\end{array}\right), \quad J_{2}=i\left(\begin{array}{cc}
0 & 1 \\
-1 & 0
\end{array}\right)
$$

In view of (2.23)-(2.28), we have

$$
\begin{aligned}
\left\|B_{j, v}\right\|_{\Theta\left(\sigma_{v+1}\right) \times \Omega}^{*} & \leqslant C(v+1)^{6 m+6}, \\
\left\|\partial_{\vartheta} B_{j, v}\right\|_{\Theta\left(\sigma_{v+1}\right) \times \Omega}^{*} & \leqslant C(v+1)^{6 m+8}, \\
\left\|\partial_{\vartheta \vartheta} B_{j, v}\right\|_{\Theta\left(\sigma_{v+1}\right) \times \Omega}^{*} & \leqslant C(v+1)^{6 m+10},
\end{aligned}
$$

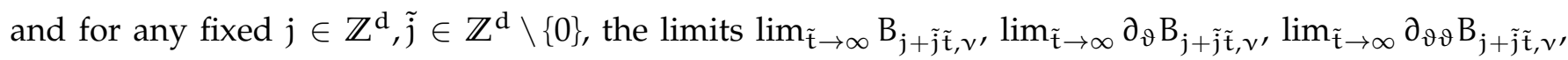
$\lim _{\tilde{\mathfrak{t}} \rightarrow \infty} \partial_{\omega} B_{\mathfrak{j}+\tilde{\mathfrak{j}}, v}, \lim _{\tilde{\mathfrak{t}} \rightarrow \infty} \partial_{\omega} \partial_{\vartheta} B_{\mathfrak{j}+\tilde{j} \tilde{\mathfrak{t}}, v}$, and $\lim _{\tilde{\mathfrak{t}} \rightarrow \infty} \partial_{\omega} \partial_{\vartheta \vartheta} B_{\mathfrak{j}+\tilde{\mathfrak{j}}, v}$ exist and

$$
\begin{array}{r}
\left\|B_{j+\tilde{j} \tilde{t}, v}-\lim _{\tilde{\mathfrak{t}} \rightarrow \infty} B_{j+\tilde{j} \tilde{t}, v}\right\|_{\Theta\left(\sigma_{v+1}\right) \times \Omega}^{*} \leqslant \frac{1}{|\tilde{t}|}(v+1)^{6 m+6}, \\
\left\|\partial_{\vartheta} B_{j+\tilde{j} \tilde{t}, v}-\lim _{\tilde{\mathfrak{t}} \rightarrow \infty} \partial_{\vartheta} B_{j+\tilde{j} \tilde{\mathfrak{t}}, v}\right\|_{\Theta\left(\sigma_{v+1}\right) \times \Omega}^{*} \leqslant \frac{1}{|\tilde{t}|}(v+1)^{6 m+8}, \\
\left\|\partial_{\vartheta \vartheta} B_{j+\tilde{j} \tilde{\mathfrak{t}}, v}-\lim _{\tilde{\mathfrak{t}} \rightarrow \infty} \partial_{\vartheta \vartheta} B_{j+\tilde{j} \tilde{\mathfrak{t}}, v}\right\|_{\Theta\left(\sigma_{v+1}\right) \times \Omega}^{*} \leqslant \frac{1}{|\tilde{t}|}(v+1)^{6 m+10} .
\end{array}
$$

Let

$$
\mathrm{B}_{v}(\vartheta ; \omega)=\left(\begin{array}{cccccccc}
\ddots & \ldots & 0 & 0 & 0 & 0 & \ldots & \cdots \\
\vdots & \ddots & 0 & 0 & 0 & 0 & \ddots & \vdots \\
\cdots & 0 & 0 & 0 & 0 & f_{j, v} & 0 & \cdots \\
\cdots & 0 & 0 & \ddots & \cdots & 0 & 0 & \ldots \\
\cdots & 0 & 0 & \ldots & \ddots & 0 & 0 & \cdots \\
\cdots & 0 & f_{j, v} & 0 & 0 & 0 & 0 & \cdots \\
\vdots & \ddots & 0 & 0 & 0 & 0 & \ddots & \vdots \\
\cdots & \ldots & 0 & 0 & 0 & 0 & \cdots & \ddots
\end{array}\right), \quad J=i\left(\begin{array}{cc}
0 & \widetilde{\mathrm{E}}_{\infty \times \infty} \\
-\widetilde{\mathrm{E}}_{\infty \times \infty} & 0
\end{array}\right),
$$

where $B_{v}(\vartheta ; \omega)$ is a matrix in which the other elements are 0 except the skew diagonal elements, and $\widetilde{\mathrm{E}}_{\infty \times \infty}$ is a matrix in which the other elements are 0 except the skew diagonal elements are 1 . Moreover, 
we note that the vector field $X_{\mathcal{F}_{v}}$ is as follows

$$
\left\{\begin{array}{l}
\dot{\vartheta}=0, \\
\frac{d}{d t}\left(\begin{array}{c}
q_{j} \\
\bar{q}_{j}
\end{array}\right)=\varepsilon_{v} \partial_{2} B_{j, v}(\vartheta ; \omega) \cdot\left(\begin{array}{c}
q_{j} \\
\bar{q}_{j}
\end{array}\right), \quad j \in \mathbb{Z}^{d}, \\
\dot{j}=\varepsilon_{v} \sum_{j \in \mathbb{Z}^{\mathrm{d}}} \partial_{\vartheta} f_{j, v}(\vartheta ; \omega) q_{j} \bar{q}_{j},
\end{array}\right.
$$

or equivalently,

$$
\left\{\begin{array}{l}
\dot{\vartheta}=0 \\
\dot{w}=\varepsilon_{v} \partial B_{v}(\vartheta ; \omega) \cdot w \\
\dot{J}=\varepsilon_{v} \sum_{j \in \mathbb{Z}^{\mathrm{d}}} \partial_{\vartheta} f_{j, v}(\vartheta ; \omega) q_{j} \bar{q}_{j}
\end{array}\right.
$$

Integrating the above equation from 0 to $t$, we get $X_{\mathcal{F}_{v}}^{t}$ :

$$
\left\{\begin{array}{l}
\vartheta=\vartheta^{\mathrm{e}}, \\
\left(\begin{array}{c}
\mathrm{q}_{j}(\mathrm{t}) \\
\bar{q}_{j}(\mathrm{t})
\end{array}\right)=\exp \left(\varepsilon_{v} \mathrm{~J}_{2} \mathrm{~B}_{j, v}\left(\vartheta^{\mathrm{e}} ; \omega\right) \mathrm{t}\right) \cdot\left(\begin{array}{c}
\mathrm{q}_{j}(0) \\
\bar{q}_{j}(0)
\end{array}\right), \quad j \in \mathbb{Z}^{\mathrm{d}}, \\
J(t)=J(0)+\int_{0}^{t} \varepsilon_{v} \sum_{j \in \mathbb{Z}^{\mathrm{d}}} \partial_{\vartheta} \mathrm{f}_{j, v}\left(\vartheta^{\mathrm{e}} ; \omega\right) \cdot \mathrm{q}_{j}(\mathrm{t}) \cdot \overline{\mathrm{q}}_{j}(\mathrm{t}) \mathrm{dt},
\end{array}\right.
$$

where $\vartheta^{\mathcal{C}}$ is a constant vector in $\mathbb{C}^{\mathrm{m}} / 2 \pi \mathbb{Z}^{\mathrm{m}}$ and $\left(\vartheta^{\mathrm{C}}, \mathrm{J}(0), \mathrm{q}(0), \overline{\mathrm{q}}(0)\right)$ is the initial value. Obviously, $J_{2} B_{j, v}(\vartheta ; \omega)$ are the diagonal matrices, thus we have $\exp \left(\varepsilon_{v} \mathcal{J}_{2} B_{j, v}\left(\vartheta^{C} ; \omega\right) t\right)$ are the diagonal matrices. That is $q_{j}(t)$ and $\bar{q}_{j}(t)$ only depend on respectively the initial value $q_{j}(0)$ and $\bar{q}_{j}(0)$. The above equation (2.30) is equivalent to

$$
\left\{\begin{array}{l}
\vartheta=\vartheta^{e} \\
w(t)=\exp \left(\varepsilon_{v} \partial B_{v}\left(\vartheta^{e} ; \omega\right) t\right) \cdot w(0), \\
J(t)=J(0)+\int_{0}^{t} \varepsilon_{v} \sum_{j \in \mathbb{Z}^{d}} \partial_{\vartheta} f_{j, v}\left(\vartheta^{e} ; \omega\right) \cdot q_{j}(t) \cdot \bar{q}_{j}(t) d t
\end{array}\right.
$$

where $\left(\vartheta^{\mathcal{C}}, J(0), w(0)\right)$ is the initial value. In view of $\varepsilon_{v}=\varepsilon^{(1+\rho)^{v}}$, we have

$$
\left|\varepsilon_{v}^{1-\rho}(\nu+1)^{6 m+10}\left(C_{*} \tilde{\rho}^{-1}\right)^{2 v}\right| \leqslant C, \quad v=0,1, \ldots
$$

as $\varepsilon<1$, where $C$ is an absolute constant independent on $v, \varepsilon$. From (2.23), we obtain, for $\vartheta \in \Theta\left(\sigma_{v+1}\right)$,

$$
\varepsilon_{v} \partial_{2} B_{j, v}(\vartheta ; \omega)=\varepsilon_{v}(v+1)^{6 m+6} B_{j, v}^{* 1}(\vartheta ; \omega)=\varepsilon_{v}^{\rho} B_{j, v}^{*}(\vartheta ; \omega),
$$

where

$$
\left\|B_{j, v}^{*}(\vartheta ; \omega)\right\|_{\Theta\left(\sigma_{v+1}\right) \times \Omega}^{*} \leqslant C,
$$

thus we have

$$
\left\|\varepsilon_{v} \partial B_{v}(\vartheta ; \omega)\right\|_{a, \Theta\left(\sigma_{v+1}\right) \times \Omega}^{*} \leqslant C \varepsilon_{v}^{\rho} .
$$

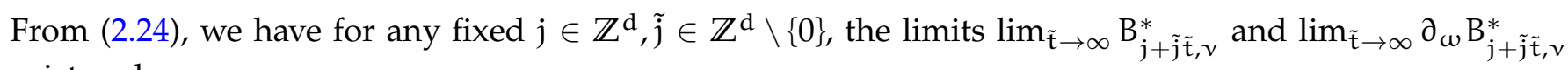
exist and

$$
\left\|B_{\mathfrak{j}+\tilde{j} \tilde{\mathfrak{t}}, v}^{*}-\lim _{\tilde{\mathfrak{t}} \rightarrow \infty} B_{\mathfrak{j}+\tilde{\mathfrak{j}} \tilde{\mathrm{t}, \nu}}^{*}\right\|_{\Theta\left(\sigma_{v+1}\right) \times \Omega}^{*} \leqslant \frac{1}{|\tilde{\mathrm{t}}|} .
$$


From (2.25), we obtain,

$$
\partial_{\vartheta}\left(\varepsilon_{v} \partial_{2} B_{j, v}(\vartheta ; \omega) \cdot\left(\begin{array}{c}
q_{j} \\
\bar{q}_{j}
\end{array}\right)\right)=\varepsilon_{v}(v+1)^{6 m+8} \cdot \partial_{\vartheta}\left(B_{j, v}^{* 1}(\vartheta ; \omega) \cdot\left(\begin{array}{c}
q_{j} \\
\bar{q}_{j}
\end{array}\right)\right)=\varepsilon_{v}^{\rho} \cdot \partial_{\vartheta}\left(B_{j, v}^{*}(\vartheta ; \omega) \cdot\left(\begin{array}{c}
q_{j} \\
\bar{q}_{j}
\end{array}\right)\right),
$$

where

$$
\left\|\partial_{\vartheta}\left(B_{j, v}^{*}(\vartheta ; \omega) \cdot\left(\begin{array}{c}
q_{j} \\
\bar{q}_{j}
\end{array}\right)\right)\right\|_{\Theta\left(\sigma_{v+1}\right) \times \Omega}^{*} \leqslant C\left(\left|q_{j}\right|+\left|\bar{q}_{j}\right|\right),
$$

thus we have

$$
\left\|\partial_{\vartheta}\left(\varepsilon_{v} \partial B_{v}(\vartheta ; \omega) \cdot w\right)\right\|_{D_{v+1}^{a} \times \Omega}^{*} \leqslant C \varepsilon_{v}^{\rho} \Gamma_{v+1} .
$$

We have for any fixed $\mathfrak{j} \in \mathbb{Z}^{\mathrm{d}}, \tilde{\mathfrak{j}} \in \mathbb{Z}^{\mathrm{d}} \backslash\{0\}$, the limits $\lim _{\tilde{\mathfrak{t}} \rightarrow \infty} \partial_{\vartheta} B_{\mathfrak{j}+\tilde{\mathfrak{j}} \tilde{\mathrm{t}}, v}^{*}$ and $\lim _{\tilde{\mathfrak{t}} \rightarrow \infty} \partial_{\omega} \partial_{\vartheta} B_{\mathfrak{j}+\tilde{\mathfrak{j}} \tilde{\mathfrak{t}}, v}$ exist from (2.27), and

$$
\left\|\partial_{\vartheta} B_{\mathfrak{j}+\tilde{\mathfrak{j}} \tilde{\mathrm{t}}, v}^{*}-\lim _{\tilde{\mathfrak{t}} \rightarrow \infty} \partial_{\vartheta} B_{\mathfrak{j}+\tilde{\mathfrak{j}} \tilde{\mathrm{t}}, v}^{*}\right\|_{\Theta\left(\sigma_{v+1}\right) \times \Omega}^{*} \leqslant \frac{1}{|\tilde{\mathrm{t}}|} .
$$

Therefor by (2.33) and (2.34), we have

$$
\exp \left(\varepsilon_{v} \partial B_{v}(\vartheta ; \omega) t\right)=I d+g_{v}^{\infty}(\vartheta ; \omega, t)
$$

where $g_{v}^{\infty}(\vartheta ; \omega, t)$ is a diagonal matrix and

$$
\left\|g_{v}^{\infty}(\vartheta ; \omega, t)\right\|_{a, \Theta\left(\sigma_{v+1}\right) \times \Omega}^{*} \leqslant C \varepsilon_{v}^{\rho}, \quad\left\|\partial_{\vartheta}\left(g_{v}^{\infty}(\vartheta ; \omega, t) \cdot w\right)\right\|_{D_{v+1}^{a} \times \Omega}^{*} \leqslant C \varepsilon_{v}^{\rho} \Gamma_{v+1}, \quad t \in[0,1] .
$$

Let

$$
g_{J, v}(\vartheta, w ; \omega, t)=\int_{0}^{t} \varepsilon_{v} \sum_{j \in \mathbb{Z}^{d}} \partial_{\vartheta} f_{j, v}(\vartheta ; \omega) \cdot q_{j}(t) \cdot \bar{q}_{j}(t) d t
$$

then

$$
J(t)=J+g_{J, v}(\vartheta, w ; \omega, t)
$$

In view of (2.25), (2.37), and (2.32), we have

$$
\left\|g_{J, v}(\vartheta, w ; \omega, t)\right\|_{D_{v+1}^{a} \times \Omega}^{*} \leqslant C \varepsilon_{v}^{\rho} \Gamma_{v}^{2}, \quad t \in[0,1],
$$

and for arbitrary $w^{\prime} \in l^{a} \times l^{a}$, we have

$$
\left\|\partial_{w}\left(g_{J, v}(\vartheta, w ; w, t)\right) \cdot w^{\prime}\right\|_{D_{v+1}^{a} \times \Omega}^{*} \leqslant C_{\varepsilon_{v}}^{\rho} \Gamma_{v} \cdot\left\|w^{\prime}\right\|_{a}, \quad t \in[0,1] .
$$

In view of (2.26), (2.37), and (2.32), we have

$$
\left\|\partial_{\vartheta}\left(g_{J, v}(\vartheta, w ; \omega, t)\right)\right\|_{D_{v+1}^{a} \times \Omega}^{*} \leqslant C \varepsilon_{v}^{\rho} \Gamma_{v}^{2}, \quad t \in[0,1] .
$$

Let

$$
X_{\mathcal{F}_{v}}^{\mathrm{t}}=\Pi_{y}+g_{v}(\omega, \mathrm{t}): \mathrm{D}_{v+1}^{\mathrm{a}} \times \Omega \mapsto \mathrm{D}_{v}^{\mathrm{a}},
$$

by (2.31), (2.36), and (2.38), we have

$$
\left\{\begin{array}{l}
\Pi_{\vartheta} \circ X_{\mathcal{F}_{v}}^{\mathrm{t}}(\vartheta, J, w)=\vartheta: \quad D_{v+1}^{\mathrm{a}} \times \Omega \mapsto \Theta\left(\sigma_{v}\right), \\
\Pi_{w} \circ X_{\mathcal{F}_{v}}^{\mathrm{t}}(\vartheta, J, w)=\left(I d+g_{v}^{\infty}(\vartheta ; \omega, \mathrm{t})\right) \cdot w: \quad D_{v+1}^{\mathrm{a}} \times \Omega \mapsto l^{\mathrm{a}} \times l^{\mathrm{a}}, \\
\Pi_{\mathrm{J}} \circ X_{\mathcal{F}_{v}}^{\mathrm{t}}(\vartheta, J, w)=J+g_{J, v}(\vartheta, w ; \omega, \mathrm{t}): \quad D_{v+1}^{\mathrm{a}} \times \Omega \mapsto \mathbb{C}^{\mathrm{m}},
\end{array}\right.
$$

where $\Pi_{y}, \Pi_{\omega}$ denote the projectors

$$
\Pi_{y}: y^{a} \times \Omega \longmapsto y^{a}, \quad \Pi_{\omega}: y^{a} \times \Omega \longmapsto \Omega,
$$


and $\Pi_{\vartheta}, \Pi_{\mathrm{J}}$, and $\Pi_{w}$ denote the projectors of $y^{a}=\mathbb{C}^{m} / 2 \pi \mathbb{Z}^{m} \times \mathbb{C}^{m} \times l^{a} \times l^{a}$ on the first, second, and third factor respectively. In view of the first equation of (2.37), (2.39), and (2.43), we get

$$
\begin{aligned}
\left|X_{\mathcal{F}_{v}}^{\mathrm{t}}-\Pi_{y}\right|_{a, D_{v+1}^{a} \times \Omega}^{*} & =\left|\left(0, g_{v}^{\infty}(\vartheta ; \omega, t) w, g_{J, v}(\vartheta, w ; \omega, t)\right)\right|_{a, D_{v+1}^{a} \times \Omega}^{*} \\
& =\frac{\left\|g_{v}^{\infty}(\vartheta ; \omega, t) w\right\|_{a, D_{v+1}^{a} \times \Omega}^{a}}{\Gamma_{v+1}}+\frac{\left\|g_{J, v}(\vartheta, w ; \omega, t)\right\|_{D_{v+1}^{a} \times \Omega}^{*}}{\Gamma_{v+1}^{2}} \\
& \leqslant \frac{\left\|g_{v}^{\infty}(\vartheta ; \omega, t)\right\|_{a, \Theta_{\sigma_{v+1}} \times \Omega}^{*} \cdot\|w\|_{a}}{\Gamma_{v+1}}+\frac{\left\|g_{J, v}(\vartheta, w ; \omega, t)\right\|_{D_{v+1}^{a} \times \Omega}^{*}}{\Gamma_{v+1}^{2}} \\
& \leqslant 3 C \varepsilon_{v}^{\rho} \leqslant C \varepsilon_{v}^{\rho},
\end{aligned}
$$

where $C:=3 C$. In view of (2.43), we have

$$
\operatorname{DX}_{\mathcal{F}_{v}}^{\mathrm{t}}=\left(\begin{array}{ccc}
\operatorname{Id}_{\mathfrak{m} \times \mathrm{m}} & 0 & 0 \\
\partial_{\vartheta}\left(g_{v}^{\infty}(\vartheta ; \omega, t) w\right) & \operatorname{Id}_{\infty \times \infty}+g_{v}^{\infty}(\vartheta ; \omega, t) & 0 \\
\partial_{\vartheta}\left(g_{J, v}(\vartheta, w ; \omega, t)\right) & \partial_{w}\left(g_{J, v}(\vartheta, w ; \omega, t)\right) & \operatorname{Id}_{m \times m}
\end{array}\right)
$$

where $\mathrm{D}$ is the differentiation operator with respect to $(\vartheta, w, J)$. In view of (2.37), (2.40), and (2.41), for $\tilde{w}=\left(\vartheta^{\prime}, w^{\prime}, J^{\prime}\right),(\vartheta, w, J) \in D_{v+1}^{a}$, we have

$$
\begin{aligned}
& \left|\left(\mathrm{DX}_{\mathcal{F}_{v}}^{\mathrm{t}}-\mathrm{Id}\right) \tilde{w}\right|_{a} \\
& =\left|\left(0, \partial_{\vartheta}\left(g_{v}^{\infty}(\vartheta ; \omega, t) w\right) \vartheta^{\prime}+g_{v}^{\infty}(\vartheta ; \omega, t) w^{\prime}, \partial_{\vartheta}\left(g_{J, v}(\vartheta, w ; \omega, t)\right) \vartheta^{\prime}+\partial_{w}\left(g_{J, v}(\vartheta, w ; \omega, t)\right) w^{\prime}\right)\right|_{a}
\end{aligned}
$$

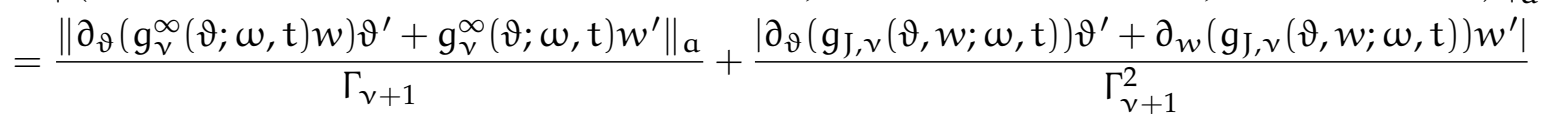

$$
\begin{aligned}
& \leqslant \frac{\mathrm{C} \varepsilon_{v}^{\rho} \Gamma_{v+1}\left|\vartheta^{\prime}\right|+\mathrm{C} \varepsilon_{v}^{\rho}\left\|w^{\prime}\right\|_{\mathrm{a}}}{\Gamma_{v+1}}+\frac{\mathrm{C} \varepsilon_{v}^{\rho} \Gamma_{v}^{2}\left|\vartheta^{\prime}\right|+\mathrm{C} \varepsilon_{v}^{\rho} \Gamma_{v}\left\|w^{\prime}\right\|_{\mathrm{a}}}{\Gamma_{v+1}^{2}} \\
& \leqslant C \varepsilon_{v}^{\rho}\left|\vartheta^{\prime}\right|+C_{\varepsilon_{v}}^{\rho} \frac{\left\|w^{\prime}\right\|_{a}}{\Gamma_{v+1}}+2 C \varepsilon_{v}^{\rho}\left|\vartheta^{\prime}\right|+2 C_{\varepsilon}^{\rho} \frac{\left\|w^{\prime}\right\|_{a}}{\Gamma_{v+1}} \\
& \leqslant 3 C \varepsilon_{v}^{\rho}|\tilde{w}|_{a} \leqslant C \varepsilon_{v}^{\rho}|\tilde{w}|_{a}
\end{aligned}
$$

where $C:=3 C$. Therefore, we have

$$
\left|\mathrm{D} X_{\mathcal{F}_{v}}^{\mathrm{t}}-\mathrm{Id}\right|_{a, D_{v+1}^{a} \times \Omega}^{\mathrm{a}}<\mathrm{C} \varepsilon_{v}^{\rho} .
$$

Similarly, we can get

$$
\left|\partial_{\omega}\left(D X_{\mathcal{F}_{v}}^{\mathrm{t}}-\mathrm{Id}\right)\right|_{\mathrm{a}, \mathrm{D}_{v+1}^{\mathrm{a}} \times \Omega}<\mathrm{C} \varepsilon_{v}^{\rho}
$$

and

$$
\left|\mathrm{D} X_{\mathcal{F}_{v}}^{\mathrm{t}}-\mathrm{Id}\right|_{\mathrm{a}, \mathrm{D}_{v+1}^{\mathrm{a}} \times \Omega}^{*}<\mathrm{C} \varepsilon_{v}^{\rho} .
$$

We now estimate the smaller terms of (2.14). Note that those terms are a polynomial of $q_{j} \bar{q}_{j}$. Thus, we can write

$$
\varepsilon_{v} \int_{0}^{1}(1-t)\left\{\left\{H_{v}^{2}, \mathcal{F}_{v}\right\}, F_{v}\right\} \circ X_{\mathcal{F}_{v}}^{\mathrm{t}} d t+\varepsilon_{v} \int_{0}^{1}\left\{R_{v}, F_{v}\right\} \circ X_{\mathcal{F}_{v}}^{\mathrm{t}} d t=\varepsilon_{v}^{2} \sum_{j \in \mathbb{Z}^{\mathrm{d}}} \tilde{R}_{j, v+1}(\vartheta ; \omega) \mathrm{q}_{j} \overline{\mathrm{q}}_{j}
$$

where by

$$
\left\{H_{v}^{2}, \mathcal{F}_{v}\right\}=\varepsilon_{v} \sum_{j \in \mathbb{Z}^{\mathrm{d}}}\left[\mathrm{R}_{j, v}\right] \mathrm{q}_{j} \overline{\mathrm{q}}_{j}-\mathrm{R}_{v}
$$


We know that $\tilde{R}_{j, v+1}(\vartheta ; \omega)$ are the linear combination of the product of $f_{j, v}(\vartheta ; \omega)$ and $R_{j, v}(\vartheta ; \omega)$. In view of $(2.23)$ and $(2.8)_{v}$ we get

$$
f_{j, v}(\vartheta ; \omega)=(v+1)^{6 m+6} f_{j, v}^{*}(\vartheta ; \omega), \quad\left\|f_{j, v}^{*}\right\|_{\Theta\left(\sigma_{v+1}\right) \times \Omega}^{*} \leqslant C
$$

and

respectively. Thus,

$$
\left\|R_{j, v}(\vartheta ; \omega)\right\|_{\Theta\left(\sigma_{v+1}\right) \times \Omega}^{*} \leqslant C,
$$

$$
\tilde{R}_{j, v+1}(\vartheta ; \omega)=(v+1)^{6 m+6} \tilde{R}_{j, v+1}^{*}(\vartheta ; \omega), \quad\left\|\tilde{R}_{j, v+1}^{*}\right\|_{\Theta\left(\sigma_{v+1}\right) \times \Omega}^{*} \leqslant C,
$$

and from (2.24) and (2.9) $)_{v}$, we have for any fixed $j \in \mathbb{Z}^{\mathrm{d}}, \tilde{\mathfrak{j}} \in \mathbb{Z}^{\mathrm{d}} \backslash\{0\}$, the limits $\lim _{\tilde{\mathfrak{t}} \rightarrow \infty} \tilde{\mathrm{R}}_{\mathfrak{j}+\tilde{j} \tilde{\mathrm{t}}, v+1}$ and $\lim _{\tilde{\mathfrak{t}} \rightarrow \infty} \partial_{\omega} \tilde{R}_{j+\tilde{j} \tilde{t}, v+1}$ exist and

$$
\left\|\tilde{R}_{\mathfrak{j}+\tilde{j} \tilde{\mathfrak{t}}, v+1}-\lim _{\tilde{\mathfrak{t}} \rightarrow \infty} \tilde{R}_{\mathfrak{j}+\tilde{j} \tilde{\mathbf{t}}, v+1}\right\|_{\Theta\left(\sigma_{v+1}\right) \times \Omega} \leqslant \frac{1}{|\tilde{\mathfrak{t}}|}(v+1)^{6 \mathfrak{m}+6} .
$$

Then

$$
R_{j, v+1}:=\varepsilon_{v}^{1-\rho} \tilde{R}_{j, v+1}, \quad\left\|R_{j, v+1}\right\|_{\Theta\left(\sigma_{v+1}\right) \times \Omega}^{*} \leqslant C
$$

because of $\varepsilon_{v}^{1-\rho}(v+1)^{6 m+6} \leqslant 1$ as $\varepsilon<1$. And we have for any fixed $j \in \mathbb{Z}^{\mathrm{d}}, \tilde{j} \in \mathbb{Z}^{\mathrm{d}} \backslash\{0\}$, the limits $\lim _{\tilde{\mathfrak{t}} \rightarrow \infty} R_{\mathfrak{j}+\tilde{j} \tilde{\mathfrak{t}}, v+1}$ and $\lim _{\tilde{\mathfrak{t}} \rightarrow \infty} \partial_{\omega} R_{\mathfrak{j}+\tilde{j} \tilde{\mathfrak{t}}, v+1}$ exist and

$$
\left\|R_{j+\tilde{j} \tilde{\mathfrak{t}}, v+1}-\lim _{\tilde{\mathfrak{t}} \rightarrow \infty} R_{\mathbf{j}+\tilde{j} \tilde{\mathfrak{j}}, v+1}\right\|_{\Theta\left(\sigma_{v+1}\right) \times \Omega} \leqslant \frac{1}{|\tilde{\mathfrak{t}}|} .
$$

In view of $\varepsilon_{v}^{2-(1-\rho)}=\varepsilon_{v+1}$, this implies $(2.7)_{v+1}$ is defined in $\mathrm{D}_{v+1}^{\mathrm{a}}$ and $\lambda_{j, v+1}$ satisfies $(2.10)_{v+1},(2.11)_{v+1}$ and $(2.12)_{v+1}$ and $R_{j, v+1}$ satisfy $(2.8)_{v+1}$ and $(2.9)_{v+1}$.

Obviously, we know

$$
\mathrm{D}_{0}^{\mathrm{a}} \supset \mathrm{D}_{1}^{\mathrm{a}} \supset \cdots \supset \mathrm{D}_{v}^{\mathrm{a}} \supset \cdots \supset \mathrm{D}_{\infty}^{\mathrm{a}}
$$

Moreover, in view of (2.42), (2.44), and (2.45), let

$$
\mathrm{S}_{v}=X_{\mathcal{F}_{v}}^{1}=\Pi_{y}+g_{v}(\omega, 1): D_{v+1}^{a} \times \Omega \longmapsto D_{v}^{a}
$$

then we have

$$
\left|S_{v}-\Pi_{y}\right|_{a, D_{v+1}^{a} \times \Omega}^{*} \leqslant C \varepsilon_{v}^{\rho}, \quad\left|D S_{v}-I d\right|_{a, D_{v+1}^{a} \times \Omega}^{*} \leqslant C \varepsilon_{v}^{\rho} .
$$

Now we are ready to prove the limiting transformation $S_{0} \circ S_{1} \circ \cdots$ converges to a transformation $\Sigma_{\infty}^{0}$ and that this transformation integrates the equation (2.5). For $\omega \in \Omega$ and $0 \leqslant r \leqslant N$ let us denote by $\Sigma_{N}^{r}$ the map

$$
\Sigma_{\mathrm{N}}^{\mathrm{r}}(\cdot ; \omega)=\mathrm{S}_{\mathrm{r}}(\cdot ; \omega) \circ \cdots \circ \mathrm{S}_{\mathrm{N}-1}(\cdot ; \omega): \mathrm{D}_{\mathrm{N}}^{\mathrm{a}} \longmapsto \mathrm{D}_{\mathrm{r}}^{\mathrm{a}}
$$

as usual, $\Sigma_{r}^{r}$ is the identity mapping. The following statement is similar to Lemmas 2.4 and 2.5 (pp. 63, 64) in [18]. For $r, l \geqslant 0$, we have

$$
\Sigma_{r+l}^{r}-\Pi_{y}=\left(S_{r}-\Pi_{y}\right) \circ\left(\Sigma_{r+l}^{r+1} \times \Pi_{\omega}\right)+\left(\Sigma_{r+l}^{r+1}-\Pi_{y}\right) .
$$

In view of (2.46), we have

$$
\begin{aligned}
& \left|\left(S_{r}-\Pi_{y}\right) \circ\left(\Sigma_{r+l}^{r+1} \times \Pi_{\omega}\right)\right|_{a, D_{r+l}^{a} \times \Omega}^{*} \\
& =\left|\left(S_{r}-\Pi_{y}\right)\left(\Sigma_{r+l}^{r+1}(\eta ; \omega) ; \omega\right)-\left(S_{r}-\Pi_{y}\right)(\eta ; \omega)+\left(S_{r}-\Pi_{y}\right)(\eta ; \omega)\right|_{a, D_{r+l}^{a} \times \Omega}^{*} \\
& \leqslant\left|\left(S_{r}-\Pi_{y}\right)\left(\Sigma_{r+l}^{r+1}(\eta ; \omega) ; \omega\right)-\left(S_{r}-\Pi_{y}\right)(\eta ; \omega)\right|_{a, D_{r+l}^{a} \times \Omega}^{*}+\left|\left(S_{r}-\Pi_{y}\right)(\eta ; \omega)\right|_{a, D_{r+l}^{a} \times \Omega}^{*} \\
& \leqslant\left|D S_{r}-I d\right|_{a, D_{r+1}^{a} \times \Omega}^{*} \cdot\left|\Sigma_{r+l}^{r+1}-\Pi_{y}\right|_{a, D_{r+l}^{a} \times \Omega}^{*}+\left|S_{r}-\Pi_{y}\right|_{a, D_{r+1}^{a} \times \Omega}^{a} \\
& \leqslant C \varepsilon_{r}^{\rho} \cdot\left|\Sigma_{r+l}^{r+1}-\Pi_{y}\right|_{a, D_{r+l}^{a} \times \Omega}^{*}+C \varepsilon_{r}^{\rho} .
\end{aligned}
$$


Let

$$
D_{r+l}^{r}=\left|\Sigma_{r+l}^{r}-\Pi_{y}\right|_{a, D_{r+l}^{a} \times \Omega^{\prime}}^{*}
$$

from (2.47) and (2.48), we can get

$$
D_{r+l}^{r}=C \varepsilon_{r}^{\rho}\left(D_{r+l}^{r+1}+1\right)+D_{r+l}^{r+1}
$$

Therefore, by induction and $\mathrm{D}_{\mathrm{r}+\mathrm{l}}^{\mathrm{r}+\mathrm{l}}=0$, we have

$$
D_{r+l}^{r}+1 \leqslant\left(D_{r+l}^{r+l}+1\right) \prod_{s=r}^{r+l-1}\left(C \varepsilon_{s}^{\rho}+1\right)=\prod_{s=r}^{r+l-1}\left(C \varepsilon_{s}^{\rho}+1\right) \leqslant 1+3 C \varepsilon_{r}^{\rho}
$$

as $\varepsilon$ small enough. Thus we have

$$
\left|\Sigma_{r+l}^{r}-\Pi_{y}\right|_{a, D_{r+l}^{a} \times \Omega}^{*} \leqslant 3 C \varepsilon_{r}^{\rho} .
$$

In view of the definition of $S_{v}$, we know

$$
D \Sigma_{r+l}^{r}=\prod_{s=r}^{r+l-1} D S_{s}
$$

And from (2.46), we have

$$
\left|\mathrm{D} \Sigma_{\mathrm{r}+\mathrm{l}}^{\mathrm{r}}-\mathrm{Id}\right|_{\mathrm{a}, \mathrm{D}_{\mathrm{r}+\mathrm{l}}^{\mathrm{a}} \times \Omega}^{*} \leqslant 3 \mathrm{C} \varepsilon_{\mathrm{r}}^{\rho}
$$

as above. Let $\eta_{0} \in D_{\infty}^{a}$ and for $s \geqslant 1$ let $\eta_{s}=\Sigma_{l+s}^{l}\left(\eta_{0} ; \omega\right)$. Then by (2.46), (2.49), and (2.50), we have

$$
\begin{aligned}
\operatorname{dist}\left(\eta_{N+1}, \eta_{N}\right)= & \operatorname{dist}\left(\Sigma_{l+N+1}^{l}\left(\eta_{0} ; \omega\right), \Sigma_{l+N}^{l}\left(\eta_{0} ; \omega\right)\right) \\
= & \left|\Sigma_{l+N+1}^{l}\left(\eta_{0} ; \omega\right)-\Sigma_{l+N}^{l}\left(\eta_{0} ; \omega\right)\right|_{a, D_{\infty}^{a} \times \Omega}^{*} \\
\leqslant & \left|\left(\Sigma_{l+N}^{l}-\Pi_{y}\right)\left(S_{l+N}\left(\eta_{0} ; \omega\right) ; \omega\right)-\left(\Sigma_{l+N}^{l}-\Pi_{y}\right)\left(\eta_{0} ; \omega\right)\right|_{a, D_{\infty}^{a} \times \Omega}^{*} \\
& +\left|\left(S_{l+N}-\Pi_{y}\right)\left(\eta_{0} ; \omega\right)\right|_{a, D_{\infty}^{a} \times \Omega}^{*} \\
\leqslant & \left|D \Sigma_{l+N}^{l}-I d\right|_{a, D_{l+N}^{a} \times \Omega}^{a} \cdot\left|S_{l+N}-\Pi_{y}\right|_{a, D_{l+N+1}^{a} \times \Omega}^{a}+\left|S_{l+N}-\Pi_{y}\right|_{a, D_{l+N+1}^{a} \times \Omega}^{a} \\
\leqslant & \left(3 C \varepsilon_{l}^{\rho}+1\right) \cdot C \varepsilon_{l+N}^{\rho} \leqslant 2 C \varepsilon_{l+N}^{\rho} .
\end{aligned}
$$

So the sequence $\left\{\eta_{s}\right\}$ is fundamental and converges to a point $\eta_{\infty} \in y^{a}$. The right hand side of (2.51) does not depend on $\eta_{0}$. So the sequence $\left\{\Sigma_{l+N}^{l}(\cdot ; \omega)\right\}$ converges uniformly in $D_{\infty}^{a}$ to an analytic map

$$
\Sigma_{\infty}^{l}(\cdot ; \omega): \mathrm{D}_{\infty}^{\mathrm{a}} \longmapsto \mathrm{D}_{\mathrm{l}}^{\mathrm{a}}
$$

which sends $\eta_{0}$ to $\eta_{\infty}$. For each $l \leqslant l_{1}<\infty$, the following relations is obviously held,

$$
\Sigma_{\mathrm{l}_{1}}^{\mathrm{l}}(\cdot ; \omega) \circ \Sigma_{\infty}^{\mathrm{l}_{1}}(\cdot ; \omega)=\Sigma_{\infty}^{\mathrm{l}}(\cdot ; \omega)
$$

From (2.49), we have the estimate

$$
\left|\Sigma_{\infty}^{l}-\Pi_{y}\right|_{a, D_{\infty}^{a} \times \Omega}^{*} \leqslant 3 C \varepsilon_{l}^{\rho}
$$

by going to the limit. This proves (i). We remark that the Hamiltonian (2.5) satisfies the conditions $(2.7)_{v^{-}}$ $(2.12)_{v}$, with $v=0$, the above iterative procedure can run repeatedly. Thus, we can get (ii). This completes the proof.

Noting that the transformation $\Sigma_{\infty}^{0}$ is linear and diagonal, and from (i) of Lemma 2.1, we get

$$
q_{j} \circ \Sigma_{\infty}^{0}=q_{j}+\varepsilon^{\rho} \tilde{g}_{j, \infty}^{*}(\vartheta ; \omega) q_{j},
$$


where

$$
\left\|\tilde{g}_{j, \infty}^{*}(\vartheta ; \omega)\right\|_{\Theta\left(\sigma_{0} / 2\right) \times \Omega}^{*} \leqslant C .
$$

And for any fixed $j \in \mathbb{Z}^{d}, \tilde{j} \in \mathbb{Z}^{d} \backslash\{0\}$, we have $\lim _{\tilde{\mathfrak{t}} \rightarrow \infty} \tilde{\mathfrak{g}}_{\mathfrak{j}+\tilde{\mathfrak{j}} \tilde{\mathrm{t}}, \infty}^{*}(\vartheta ; \omega)$ and $\lim _{\tilde{\mathfrak{t}} \rightarrow \infty} \partial_{\omega} \tilde{g}_{\mathfrak{j}+\tilde{\mathfrak{j}} \tilde{\mathrm{t}}, \infty}^{*}(\vartheta ; \omega)$ exist and

$$
\left\|\tilde{g}_{\mathfrak{j}+\tilde{j} \tilde{\mathfrak{t}}, \infty}^{*}-\lim _{\tilde{\mathfrak{t}} \rightarrow \infty} \tilde{g}_{\mathfrak{j}+\tilde{\mathfrak{j}}, \infty}^{*}\right\|_{\Theta\left(\sigma_{0} / 2\right) \times \Omega}^{*} \leqslant \frac{1}{|\tilde{\mathfrak{t}}|} .
$$

Hence, by (ii) of Lemma 2.1, Hamiltonian (2.5) is changed into by $\Sigma_{\infty}^{0}$

$$
\mathrm{H}_{0}:=\overline{\mathrm{H}} \circ \Sigma_{\infty}^{0}=<\omega, \mathrm{J}>+\sum_{j \in \mathbb{Z}^{\mathrm{d}}} \mu_{j} q_{j} \overline{\mathrm{q}}_{j}
$$

and Hamiltonian (2.3) is changed into

$$
\tilde{\mathrm{G}}=\widehat{\mathrm{G}} \circ \Sigma_{\infty}^{0}=\sum_{\alpha, \beta,|\alpha|+|\beta| \geqslant 4, \sum_{j \in \mathbb{Z}^{d}}\left(\alpha_{j}-\beta_{j}\right) j=0} \tilde{\mathrm{G}}_{\alpha \beta}(\vartheta ; \omega) q^{\alpha} \bar{q}^{\beta},
$$

where

$$
\tilde{\mathrm{G}}_{\alpha \beta}(\vartheta ; \omega)=\widehat{\mathrm{G}}_{\alpha \beta}(\vartheta)\left(1+\varepsilon^{\rho} \widehat{\mathrm{G}}_{\alpha \beta}^{*}(\vartheta ; \omega)\right), \quad\left\|\widehat{\mathrm{G}}_{\alpha \beta}^{*}(\vartheta ; \omega)\right\|_{\Theta\left(\sigma_{0} / 2\right) \times \Omega}^{*} \leqslant \mathrm{C} .
$$

And from (2.52), we have for any fixed $i, j \in \mathbb{Z}^{\mathrm{d}}$ and $\tilde{i}, \tilde{j} \in \mathbb{Z}^{\mathrm{d}} \backslash\{0\}$, if $\alpha_{\boldsymbol{i}+\tilde{i} \tilde{\mathfrak{t}}} \geqslant 1$ and $\alpha_{\mathfrak{j}+\tilde{j} \tilde{\mathrm{t}}} \geqslant 1$, denote

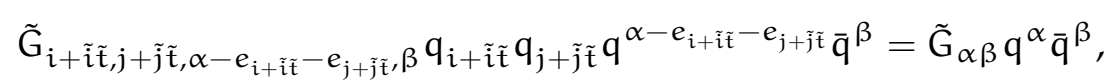

where $e_{i}$ is a vector in which the other elements are 0 except the ith element is 1 , then the limits

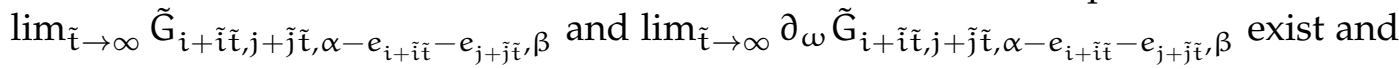

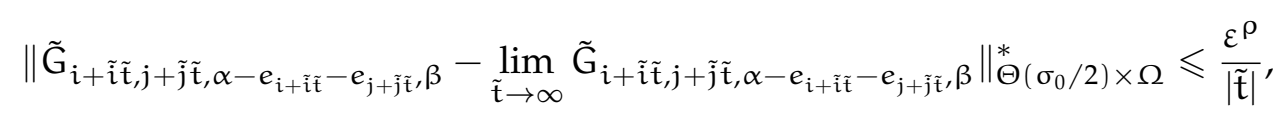

similarly, if $\alpha_{\mathfrak{i}+\tilde{i} \tilde{\mathfrak{t}}} \geqslant 1$ and $\beta_{\mathfrak{j}+\tilde{j} \tilde{\mathfrak{t}}} \geqslant 1$, denote

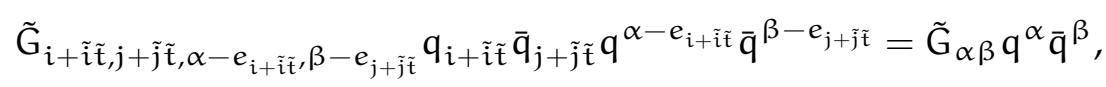

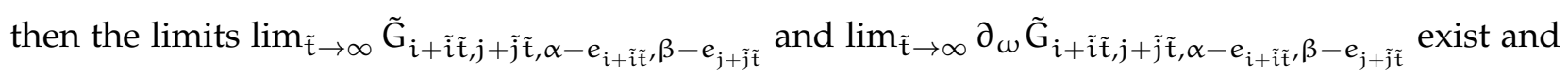

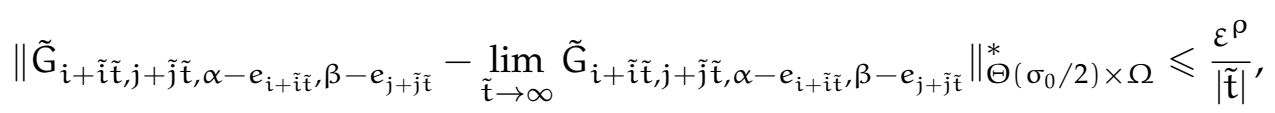

if $\beta_{i+\tilde{i} \tilde{t}} \geqslant 1$ and $\beta_{j+\tilde{j} \tilde{t}} \geqslant 1$, denote

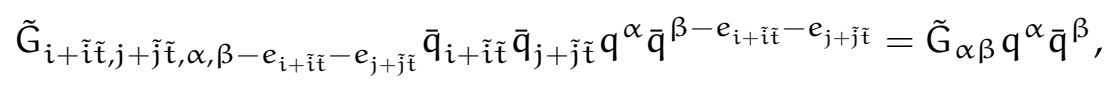

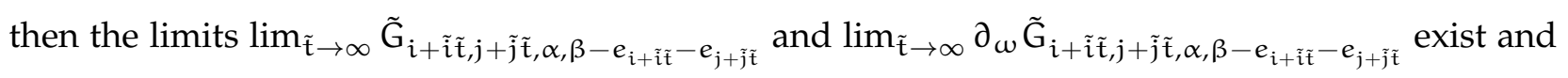

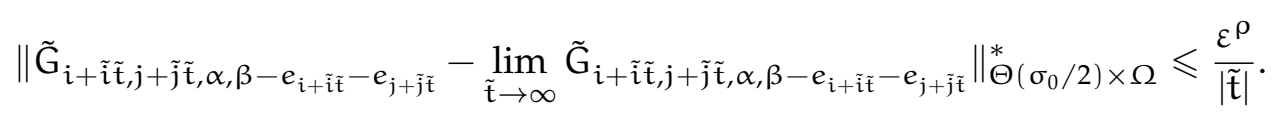

This implies the Hamiltonian (2.6) is changed by the transformation $\Sigma_{\infty}^{0}$ into

$$
\mathrm{H}=\mathrm{H}_{0}+\varepsilon \tilde{\mathrm{G}} .
$$

As in [22], we can prove the following lemma. 
Lemma 2.2. For $\mathrm{a} \geqslant 0$, the gradients $\tilde{\mathrm{G}}_{\mathrm{q}}, \tilde{\mathrm{G}}_{\overline{\mathrm{q}}}$ are real analytic for real argument as maps from some neighborhood of origin in $\mathrm{l}^{\mathrm{a}} \times \mathrm{l}^{\mathrm{a}}$ into $\mathrm{l}^{\mathrm{a}}$, with $\left\|\tilde{\mathrm{G}}_{\mathrm{q}}\right\|_{\mathrm{a}}=\mathrm{O}\left(\|\mathrm{q}\|_{\mathrm{a}}^{3}\right),\left\|\tilde{\mathrm{G}}_{\overline{\mathrm{q}}}\right\|_{\mathrm{a}}=\mathrm{O}\left(\|\mathrm{q}\|_{\mathrm{a}}^{3}\right)$, that is, there is a constant $\mathrm{C}$ such that

$$
\left\|\tilde{G}_{q}\right\|_{a} \leqslant C\|q\|_{a}^{3}, \quad\left\|\tilde{G}_{\bar{q}}\right\|_{a} \leqslant C\|q\|_{a}^{3}
$$

uniformly for $(\vartheta, \omega) \in \Theta\left(\sigma_{0} / 2\right) \times \Omega$. The Hamiltonian $\tilde{\mathrm{G}}$ depends on the "time" $\vartheta=\left(\omega_{1} \mathrm{t}, \ldots, \omega_{\mathrm{m}} \mathrm{t}\right)$ and parameter $\omega=\left(\omega_{1}, \ldots, \omega_{m}\right)$.

Proof. Due to (2.53), then for $(\vartheta, \omega) \in \Theta\left(\sigma_{0} / 2\right) \times \Omega$,

$$
\frac{\partial \tilde{\mathrm{G}}}{\partial q_{i}}=\sum_{\alpha-e_{i}, \beta,\left|\alpha-e_{i}\right|+|\beta| \geqslant 3, \Sigma_{j}\left(\alpha_{j}-\beta_{j}\right) j=0} \tilde{G}_{\alpha \beta}(\vartheta, \omega) q^{\alpha-e_{i}} \bar{q}^{\beta} .
$$

From (2.4) and (2.54), we have

$$
\begin{aligned}
\left\|\tilde{G}_{q}\right\|_{a}=\sum_{i \in \mathbb{Z}^{d}}\left|\tilde{G}_{q_{i}}\right| e^{|i| a} & \leqslant C \sum_{i \in \mathbb{Z}^{d} \alpha-e_{i}, \beta,\left|\alpha-e_{i}\right|+|\beta| \geqslant 3, \sum_{j}\left(\alpha_{j}-\beta_{j}\right) j=0}\left|q^{\alpha-e_{i}} \bar{q}^{\beta}\right| e^{\left|\left(\alpha_{i}-1-\beta_{i}\right) i+\sum_{j \neq i}\left(\alpha_{j}-\beta_{j}\right) j\right| a} \\
& \leqslant C \sum_{i \in \mathbb{Z}^{d} \alpha-e_{i}, \beta,\left|\alpha-e_{i}\right|+|\beta| \geqslant 3, \sum_{j}\left(\alpha_{j}-\beta_{j}\right) j=0}\left|q^{\alpha-e_{i}}\right| e^{\left|\left(\alpha_{i}-1\right) i+\sum_{j \neq i} j \alpha_{j}\right| a}\left|\bar{q}^{\beta}\right| e^{\left|\sum_{j} j \beta_{j}\right| a} \\
& \leqslant C\|q\|_{a}^{|\alpha|-1}\|\bar{q}\|_{a}^{\beta \mid} \leqslant C\|q\|_{a}^{|\alpha|+|\beta|-1} \leqslant C\|q\|_{a}^{3}
\end{aligned}
$$

as required. Similarly, we have $\left\|\tilde{G}_{\bar{q}}\right\|_{a} \leqslant C\|q\|_{a}^{3}$. This completes the proof.

\section{Partial Birkhoff normal form}

Next we transform the Hamiltonian (2.56) into some partial Birkhoff normal form by introducing the action-angle variable so that it may serve as a small perturbation of some integrable system in a sufficiently small neighborhood of the origin.

For given $n$ vectors in $\mathbb{Z}^{\mathrm{d}}$, say $S=\left\{i_{1}, \cdots, i_{n}\right\}$, we denote $\mathbb{Z}_{1}^{\mathrm{d}}=\mathbb{Z}^{\mathrm{d}} \backslash \mathrm{S}$. We introduce the action-angle variable by setting

$$
q_{j}= \begin{cases}\sqrt{\mathrm{I}_{j}} e^{-i \theta_{j}}, & j \in S \\ z_{j}, & j \in \mathbb{Z}_{1}^{\mathrm{d}} .\end{cases}
$$

By the symplectic change (3.1), the Hamiltonian equation (2.56) becomes

$$
\mathrm{H}_{0}+\varepsilon \tilde{\mathrm{G}}=<\omega, \mathrm{J}>+\sum_{j \in S} \mu_{j} \mathrm{I}_{j}+\sum_{j \in \mathbb{Z}_{1}^{\mathrm{d}}} \mu_{j} z_{j} \bar{z}_{j}+\mathrm{P}(\vartheta, \theta, \mathrm{J}, \mathrm{I}, z, \bar{z} ; \omega, \xi) .
$$

Denote

$$
N=<\omega, J>+\sum_{j \in S} \mu_{j} I_{j}+\sum_{j \in \mathbb{Z}_{1}^{d}} \mu_{j} z_{j} \bar{z}_{j}
$$

Hence, the total Hamiltonian (2.56) is

$$
\mathrm{H}=\mathrm{N}+\mathrm{P},
$$

where $P$ is just $\varepsilon \tilde{G}$ with the $\left(\vartheta, J, q_{i_{1}}, \cdots, q_{i_{n}}, \bar{q}_{i_{1}}, \cdots, \bar{q}_{i_{n}}, q_{j}, \bar{q}_{j}\right)$-variables expressed in terms of the $\left(\vartheta, \mathrm{J}, \theta, \mathrm{I}, z_{\mathfrak{j}}, \bar{z}_{\mathfrak{j}}\right)$-variables. 
Next, we will give out the estimates of the perturbed term P. To this end we need some notations which are taken from [17]. Let $l^{\mathrm{a}}$ be now the Hilbert space of all complex sequences $w=\left(\ldots, w_{j}, \ldots\right)_{\mathbf{j} \in \mathbb{Z}_{1}^{\mathrm{d}}}$ with

$$
\|w\|_{a}=\sum_{j \in \mathbb{Z}_{1}^{\mathrm{d}}}\left|w_{j}\right| e^{\mathrm{a}|j|}<\infty, \quad a>0 .
$$

Set $x=\vartheta \oplus \theta$ with $\theta=\left(\theta_{j}\right)_{j \in S}, y=J \oplus I, z=\left(z_{j}\right)_{j \in \mathbb{Z}_{1}^{d}}$ and $\zeta=\omega \oplus\left(\xi_{j}\right)_{j \in S}$, and let us introduce the phase space

$$
\mathcal{P}^{\mathrm{a}}=\widehat{\mathbb{T}}^{\mathrm{m}+\mathrm{n}} \times \mathbb{C}^{\mathrm{m}+\mathrm{n}} \times \mathrm{l}^{\mathrm{a}} \times \mathrm{l}^{\mathrm{a}} \ni(x, y, z, \bar{z}),
$$

where $\widehat{\mathbb{T}}^{m+n}$ is the complexiation of the usual $(m+n)$-torus $\mathbb{T}^{m+n}$. Set

$$
\mathrm{D}_{\mathrm{a}}\left(\mathrm{s}^{\prime}, \mathrm{r}\right):=\left\{(x, y, z, \bar{z}) \in \mathcal{P}^{\mathrm{a}}:|\operatorname{Im} x|<\mathrm{s}^{\prime},|y|<\mathrm{r}^{2},\|z\|_{\mathrm{a}}+\|\bar{z}\|_{\mathrm{a}}<\mathrm{r}\right\} .
$$

We define the weighted phase norms

$$
|\mathrm{W}|_{\mathrm{r}}=|x|+\frac{1}{\mathrm{r}^{2}}|y|+\frac{1}{\mathrm{r}}\|z\|_{\mathrm{a}}+\frac{1}{\mathrm{r}}\|\bar{z}\|_{\mathrm{a}}
$$

for $W=(x, y, z, \bar{z}) \in \mathcal{P}^{a}$. Denote by $\underline{\Sigma}$ the parameter set $\Omega \times[0,1]^{n}$. For a map $U: D\left(s^{\prime}, r\right) \times \underline{\Sigma} \rightarrow \mathcal{P}^{a}$, define its Lipschitz semi-norm $|\mathrm{U}|_{\mathrm{r}}^{\mathcal{L}}$ :

$$
|\mathrm{U}|_{\mathrm{r}}^{\mathcal{L}}=\sup _{\zeta \neq \zeta^{\prime}} \frac{\left|\Delta_{\zeta \zeta^{\prime}} \mathrm{U}\right|_{\mathrm{r}}}{\left|\zeta-\zeta^{\prime}\right|}
$$

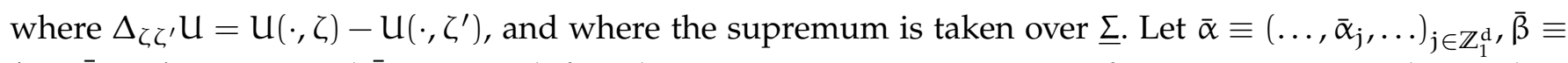
$\left(\ldots, \bar{\beta}_{j}, \ldots\right)_{j \in \mathbb{Z}_{1}^{d}}, \bar{\alpha}_{j}$ and $\bar{\beta}_{j} \in \mathbb{N}$ with finitely many nonzero components of positive integers. The product $z^{\bar{\alpha}} \bar{z}^{\bar{\beta}}$ denotes $\prod_{j} z_{j}^{\bar{\alpha}_{j}} \bar{z}_{j}^{\bar{\beta}_{j}}$. Let

$$
\mathrm{P}(x, y, z, \bar{z})=\sum_{\bar{\alpha}, \bar{\beta}} P_{\bar{\alpha} \bar{\beta}}(x, y) z^{\bar{\alpha}} \bar{z}^{\bar{\beta}},
$$

where $P_{\bar{\alpha} \bar{\beta}}=\sum_{k, b} P_{k b \bar{\alpha} \bar{\beta}} y^{b} e^{i<k, x>}$ are $C_{W}^{1}$ functions in parameter $\zeta$ in the sense of Whitney. Define the weighted norm of $P$ by

$$
\|\mathrm{P}\|_{D_{a}\left(s^{\prime}, r\right), \underline{\Sigma}} \equiv \sup _{\|z\|_{a}<r,\|\bar{z}\|_{a}<r} \sum_{\bar{\alpha}, \bar{\beta}}\left\|P_{\bar{\alpha} \bar{\beta}}\right\|\left|z^{\bar{\alpha}} \| \bar{z}^{\bar{\beta}}\right|,
$$

where, if $\mathrm{P}_{\bar{\alpha} \bar{\beta}}=\sum_{k \in \mathbb{Z}^{m+n}, b \in \mathbb{N}^{m+n}} P_{k b \bar{\alpha} \bar{\beta}}(\zeta) y^{b} e^{i<k, x>}, P_{\bar{\alpha} \bar{\beta}}$ is short for

$$
\left\|\mathrm{P}_{\bar{\alpha} \bar{\beta}}\right\| \equiv \sum_{k, \mathrm{~b}}\left|\mathrm{P}_{\mathrm{kb} \bar{\alpha} \bar{\beta}}\right| \underline{\Sigma} \mathrm{r}^{2|b|} e^{|k| s^{\prime}}, \quad\left|\mathrm{P}_{\mathrm{kb} \bar{\alpha} \bar{\beta}}\right|_{\underline{\Sigma}} \equiv \sup _{\zeta \in \underline{\Sigma}}\left(\left|\mathrm{P}_{\mathrm{kb} \bar{\alpha} \bar{\beta}}\right|+\left|\partial_{\zeta} \mathrm{P}_{\mathrm{kb} \bar{\alpha} \bar{\beta}}\right|\right)
$$

the derivative with respect to $\zeta$ is in the sense of Whitney. Denote by $X_{P}$ the vector field corresponding the Hamiltonian $P$ with respect to the symplectic structure $d x \wedge d y+i d z \wedge d \bar{z}$, namely,

$$
X_{\mathrm{P}}=\left(\partial_{y} \mathrm{P},-\partial_{x} \mathrm{P}, \nabla_{\bar{z}} \mathrm{P},-\nabla_{z} \mathrm{P}\right) .
$$

Its weighted norm is defined by

$$
\left\|X_{P}\right\|_{D_{a}\left(s^{\prime}, r\right), \underline{\Sigma}} \equiv\left\|P_{y}\right\|_{D_{a}\left(s^{\prime}, r\right), \underline{\Sigma}}+\frac{1}{r^{2}}\left\|P_{x}\right\|_{D_{a}\left(s^{\prime}, r\right), \underline{\Sigma}}+\frac{1}{r}\left(\sum_{j \in \mathbb{Z}_{1}^{d}}\left\|P_{z_{j}}\right\|_{D_{a}\left(s^{\prime}, r\right), \underline{\Sigma}} e^{|j| a}+\sum_{j \in \mathbb{Z}_{1}^{d}}\left\|P_{\bar{z}_{j}}\right\|_{D_{a}\left(s^{\prime}, r\right), \underline{\Sigma}} e^{|j| a}\right) .
$$

Lemma 3.1. The perturbation $\mathrm{P}(x, y, z, \bar{z} ; \zeta)$ is real analytic for real argument $(x, y, z, \bar{z}) \in D_{a}\left(s^{\prime}, r\right)$ for some given $\mathrm{s}^{\prime}, \mathrm{r}>0$, and Lipschitz in the parameters $\zeta \in \underline{\Sigma}$, and for each $\zeta \in \underline{\Sigma}$ its gradients with respect to $z, \bar{z}$ satisfy

$$
\partial_{z} \mathrm{P}, \quad \partial_{\bar{z}} \mathrm{P} \in \mathcal{A}\left(\mathrm{l}^{\mathrm{a}}, \mathrm{l}^{\mathrm{a}}\right),
$$


where $\mathcal{A}\left(l^{\mathrm{a}}, \mathrm{l}^{\mathrm{a}}\right)$ denotes the class of all maps from some neighborhood of the origin in $\mathrm{l}^{\mathrm{a}}$ into $\mathrm{l}^{\mathrm{a}}$, which is real analytic in the real and imaginary parts of the complex coordinate $z$. In addition, for the perturbed term $\mathrm{P}$ we have the following estimates

$$
\left\|X_{P}\right\|_{D_{a}\left(s^{\prime}, r\right), \underline{\Sigma}} \leqslant C \varepsilon, \quad\left\|\partial_{\zeta} X_{P}\right\|_{D_{a}\left(s^{\prime}, r\right), \underline{\Sigma}} \leqslant C \varepsilon,
$$

where $\mathrm{s}^{\prime}=\sigma_{0} / 2$ and $\mathrm{r}=\varepsilon^{1 / 2}$.

Proof. From (3.1) and $\|z\|_{a} \leqslant r=\varepsilon^{1 / 2}$, we get $\|q\|_{a} \leqslant C \varepsilon^{1 / 2}$ where $q=\underline{q} \oplus z$ with $q$ And $|\mathrm{P}|=|\varepsilon \tilde{G}|=\mathrm{O}\left(\varepsilon^{3}\right)$ on $\mathrm{D}\left(\mathrm{s}^{\prime}, 2 \mathrm{r}\right)$. Using Cauchy estimates for $\partial_{x} \mathrm{P}, \partial_{y} \mathrm{P}, \bar{\partial}_{\bar{z}} \mathrm{P}$ and $\partial_{z} \mathrm{P}$, we obtain $\left|\partial_{x} \mathrm{P}\right|=$ $\mathrm{O}\left(\varepsilon^{3}\right),\left|\partial_{y} \mathrm{P}\right|=\mathrm{O}\left(\varepsilon^{2}\right),\left|\partial_{\bar{z}} \mathrm{P}\right|=\mathrm{O}\left(\varepsilon^{5 / 2}\right),\left|\partial_{z} \mathrm{P}\right|=\mathrm{O}\left(\varepsilon^{5 / 2}\right)$ on $\mathrm{D}\left(\mathrm{s}^{\prime}, \mathrm{r}\right)$. Hence, we have $\left\|\mathrm{X}_{\mathrm{P}}\right\|_{\mathrm{D}_{\mathrm{a}}\left(\mathrm{s}^{\prime}, \mathrm{r}\right), \underline{\Sigma}} \leqslant \mathrm{C} \varepsilon$. Using again Cauchy estimates with respect to $\zeta$, we also have $\left\|\partial_{\zeta} X_{P}\right\|_{D_{a}\left(s^{\prime}, r\right), \underline{\Sigma}} \leqslant C \varepsilon$.

\section{An infinite-dimensional KAM theorem}

In order to prove our main result (Theorem 1.1), we need to state a KAM theorem which was proved by Geng-You [17]. Here we recite the theorem from [17].

Let us consider the perturbations of a family of Hamiltonian

$$
N=\sum_{j \in S} \widehat{\omega}_{j}(\xi) y_{j}+\sum_{j \in \mathbb{Z}_{1}^{d}} \widehat{\Omega}_{j}(\xi) z_{j} \bar{z}_{j}
$$

in $n$-dimensional angle-action coordinates $(x, y)$ and infinite-dimensional coordinates $(z, \bar{z})$ with symplectic structure

$$
\sum_{j \in S} d x_{j} \wedge d y_{j}+i \sum_{j \in \mathbb{Z}_{1}^{d}} d z_{j} \wedge d \bar{z}_{j}
$$

The tangent frequencies $\widehat{\omega}=\left(\widehat{\omega}_{j}\right)_{j \in S}$ and normal ones $\widehat{\Omega}=\left(\widehat{\Omega}_{j}\right)_{j \in \mathbb{Z}_{1}^{d}}$ depend on $n$ parameters

$$
\xi \in \Pi \subset \mathbb{R}^{n},
$$

with $\Pi$ a closed bounded set of positive Lebesgue measure.

For each $\xi$ there is an invariant $n$-torus $\mathcal{T}_{0}^{\mathfrak{n}}=\mathbb{T}^{\mathfrak{n}} \times\{0,0,0\}$ with frequencies $\widehat{\omega}(\xi)$. In its normal space described by the $z \bar{z}$-coordinates the origin is an elliptic fixed point. Hence $\mathcal{T}_{0}^{n}$ is linear stable. The aim is to prove the persistence of a large portion of this family of linearly stable rotational tori under small perturbations $\mathrm{H}=\mathrm{N}+\mathrm{P}$ of $\mathrm{N}$. To this end the following assumptions are made.

Assumption 4.1 (Non-degeneracy). The map $\xi \mapsto \widehat{\omega}(\xi)$ is a $C_{W}^{1}$ diffeomorphism between $\Pi$ and its image.

Assumption 4.2 (Asymptotics of normal frequencies).

$$
\widehat{\Omega}_{j}=|j|^{2}+\widetilde{\Omega}_{j},
$$

where $\widetilde{\Omega}_{j}^{\prime}$ s are $C_{W}^{1}$ functions of $\xi$ with $C_{W}^{1}$-norm bounded by some small positive constant $L$.

Assumption 4.3 (Melnikov's non-resonance conditions). There exist $\gamma^{\prime}, \tau>0$ such that

$$
\begin{aligned}
|<k, \widehat{\omega}>| \geqslant \frac{\gamma^{\prime}}{|k|^{\tau}}, \quad k \neq 0, & \left|<k, \widehat{\omega}>+\widehat{\Omega}_{j}\right| \geqslant \frac{\gamma^{\prime}}{|k|^{\prime}} \\
\left|<k, \widehat{\omega}>+\widehat{\Omega}_{j}+\widehat{\Omega}_{l}\right| \geqslant \frac{\gamma^{\prime}}{|k|^{\prime}}, & \left|<k, \widehat{\omega}>+\widehat{\Omega}_{j}-\widehat{\Omega}_{l}\right| \geqslant \frac{\gamma^{\prime}}{|k|^{\tau}}, \quad|k|+|| j|-| l|| \neq 0 .
\end{aligned}
$$

Assumption 4.4 (Regularity). $P$ is real analytic in $x, y, z, \bar{z}$ and Whitney smooth in $\xi$; in addition

$$
\left\|X_{P}\right\|_{D_{a}\left(s^{\prime}, r\right), \Pi}<\infty
$$


Assumption 4.5 (Special form). P admits a special form of the following

$$
\mathcal{D}=\left\{\mathrm{P}: \mathrm{P}=\sum_{k \in \mathbb{Z}^{\mathrm{n}}, \mathrm{b} \in \mathbb{N}^{\mathrm{n}}, \bar{\alpha}, \bar{\beta}} \mathrm{P}_{k \mathrm{~b} \bar{\alpha} \bar{\beta}}(\xi) y^{\mathrm{b}} e^{\mathrm{i}<k, x>} z^{\bar{\alpha}} \bar{z}^{\bar{\beta}}\right\},
$$

where $k, \bar{\alpha}, \bar{\beta}$ have the following relation

$$
\sum_{s=1}^{n} k_{s} i_{s}+\sum_{j \in \mathbb{Z}_{1}^{d}}\left(\bar{\alpha}_{j}-\bar{\beta}_{j}\right) j=0 .
$$

Assumption 4.6 (Töplitz-Lipschitz property). For any fixed $j, l \in \mathbb{Z}^{\mathrm{d}}, \tilde{\mathrm{c}} \in \mathbb{Z}^{\mathrm{d}} \backslash\{0\}$, the limits

$$
\lim _{\tilde{\mathfrak{t}} \rightarrow \infty} \widetilde{\Omega}_{\mathfrak{j}+\tilde{\mathbf{c}} \tilde{\mathfrak{t}}}, \quad \lim _{\tilde{\mathfrak{t}} \rightarrow \infty} \frac{\partial^{2} \mathrm{P}}{\partial z_{\mathfrak{j}+\tilde{\mathbf{c}} \tilde{\mathfrak{t}}} \partial z_{l-\tilde{\mathbf{c}} \tilde{\mathfrak{t}}}}, \quad \lim _{\tilde{\mathfrak{t}} \rightarrow \infty} \frac{\partial^{2} P}{\partial z_{j+\tilde{c} \tilde{\mathfrak{t}}} \partial \bar{z}_{l+\tilde{c} \tilde{\mathfrak{t}}}}, \quad \lim _{\tilde{\mathfrak{t}} \rightarrow \infty} \frac{\partial^{2} P}{\partial \bar{z}_{j+\tilde{\mathfrak{c}} \tilde{\mathfrak{t}}} \partial \bar{z}_{l-\tilde{\mathbf{c}} \tilde{\mathfrak{t}}}}
$$

exist. Moreover, there exists $K>0$, such that when $|\tilde{t}|>K, N+P$ satisfies

$$
\begin{aligned}
& \left|\widetilde{\Omega}_{\mathfrak{j}+\tilde{\mathbf{c}} \tilde{\mathfrak{t}}}-\lim _{\tilde{\mathfrak{t}} \rightarrow \infty} \widetilde{\Omega}_{\mathbf{j}+\tilde{\mathbf{c}} \tilde{\mathfrak{t}}}\right|_{\Pi} \leqslant \frac{\varepsilon}{|\tilde{\mathfrak{t}}|^{\prime}} \\
& \left\|\frac{\partial^{2} P}{\partial z_{j+\tilde{\mathbf{c}} \tilde{\mathfrak{t}}} \partial z_{l-\tilde{\mathbf{c}} \tilde{\mathfrak{t}}}}-\lim _{\tilde{\mathfrak{t}} \rightarrow \infty} \frac{\partial^{2} P}{\partial z_{\mathfrak{j}+\tilde{\mathbf{c}} \tilde{\mathfrak{t}}} \partial z_{l-\tilde{\mathbf{c}} \tilde{\mathfrak{t}}}}\right\|_{D_{a}\left(s^{\prime}, r\right), \Pi} \leqslant \frac{\varepsilon}{|\tilde{t}|} e^{-|j+l| a}, \\
& \left\|\frac{\partial^{2} P}{\partial z_{j+\tilde{c} \tilde{\mathfrak{t}}} \partial \bar{z}_{l+\tilde{c} \tilde{\mathfrak{t}}}}-\lim _{\tilde{\mathfrak{t}} \rightarrow \infty} \frac{\partial^{2} P}{\partial z_{j+\tilde{c} \tilde{\mathfrak{t}}} \partial \bar{z}_{l+\tilde{c} \tilde{\mathfrak{t}}}}\right\|_{D_{a}\left(s^{\prime}, r\right), \Pi} \leqslant \frac{\varepsilon}{|\tilde{t}|} e^{-|j-l| a}, \\
& \left\|\frac{\partial^{2} P}{\partial \bar{z}_{j+\tilde{c} \tilde{\mathfrak{t}}} \partial \bar{z}_{l-\tilde{c} \tilde{\mathfrak{t}}}}-\lim _{\tilde{\mathfrak{t}} \rightarrow \infty} \frac{\partial^{2} P}{\partial \bar{z}_{j+\tilde{c} \tilde{\mathfrak{t}}} \partial \bar{z}_{l-\tilde{c} \tilde{\mathfrak{t}}}}\right\|_{D_{a}\left(s^{\prime}, r\right), \Pi} \leqslant \frac{\varepsilon}{|\tilde{t}|} e^{-|j+l| a} .
\end{aligned}
$$

We can now state the basic KAM theorem which is attributed to Geng-You [17].

Theorem 4.7 ([17, Theorem 2]). Assume that the Hamiltonian $\mathrm{H}=\mathrm{N}+\mathrm{P}$ satisfies Assumptions 4.1-4.6. Let $\gamma^{\prime}>0$ be small enough, there exists a positive constant $\varepsilon=\varepsilon\left(\mathrm{n}, \mathrm{d}, \mathrm{K}, \tau, \gamma^{\prime}, \mathrm{s}^{\prime}, \mathrm{r}, \mathrm{a}\right)$, such that if $\left\|\mathrm{X}_{\mathrm{P}}\right\|_{\mathrm{D}_{\mathrm{a}}\left(\mathrm{s}^{\prime}, \mathrm{r}\right), \Pi}<\varepsilon$, then the following holds true: there exist a Cantor subset $\Pi_{\gamma^{\prime}} \subset \Pi$ with meas $\left(\Pi \backslash \Pi_{\gamma^{\prime}}\right)=\mathrm{O}\left(\gamma^{\prime}\right)$ and two maps (analytic in $x$ and $\mathrm{C}_{W}^{1}$ in $\xi$ )

$$
\Psi: \mathbb{T}^{\mathrm{n}} \times \Pi_{\gamma^{\prime}} \rightarrow \mathrm{D}_{\mathrm{a}}\left(\mathrm{s}^{\prime}, \mathrm{r}\right), \quad \tilde{\omega}: \Pi_{\gamma^{\prime}} \rightarrow \mathbb{R}^{\mathrm{n}},
$$

where $\Psi$ is $\frac{\varepsilon}{\left(\gamma^{\prime}\right)^{2}}$-close to the trivial embedding $\Psi_{0}: \mathbb{T}^{n} \times \Pi \rightarrow \mathbb{T}^{n} \times\{0,0,0\}$ and $\tilde{\omega}$ is $\varepsilon$-close to the unperturbed frequency $\widehat{\omega}$. Then for any $\xi \in \Pi_{\gamma^{\prime}}$ and $x \in \mathbb{T}^{n}$, the curve $t \rightarrow \Psi(x+\tilde{\omega}(\xi) t, \xi)$ is a quasi-periodic solution of the Hamiltonian equations governed by $\mathrm{H}=\mathrm{N}+\mathrm{P}$. Moreover, the obtained solutions are real analytic and linearly stable.

In order to apply the above theorem to our problem, we need to introduce a new parameter $\bar{\omega}$ below.

For fixed $\omega_{-} \in \Omega$ arbitrarily and for $\omega \in \overline{\bar{\Omega}}:=\left\{\omega \in \Omega|| \omega-\omega_{-} \mid \leqslant \varepsilon\right\}$, we can introduce new parameter $\bar{\omega}$ by the following

$$
\omega=\omega_{-}+\varepsilon \bar{\omega}, \quad \bar{\omega} \in[0,1]^{m} .
$$

Hence, the Hamiltonian (3.2) becomes

$$
H=<\widehat{\omega}(\zeta), \hat{y}>+<\widehat{\Omega}(\zeta), \hat{z}>+P
$$

where $\widehat{\omega}(\zeta)=\omega \oplus \breve{\omega}, \zeta=\bar{\omega} \oplus \xi, \hat{z}=\left(\left|z_{j}\right|^{2}\right)_{j \in \mathbb{Z}_{1}^{d}}, \hat{x}=\vartheta \oplus \theta, \hat{y}=J \oplus$ I with

$$
\begin{array}{ll}
\breve{\omega}_{i}=\mu_{i}, & i \in S, \\
\widehat{\Omega}_{j}=\mu_{j}, & j \in \mathbb{Z}_{1}^{d} .
\end{array}
$$

Denote $\breve{\omega}(\zeta)=\left(\ldots, \mu_{i}, \ldots\right)_{i \in S}, \quad \widehat{\Omega}(\zeta)=\left(\ldots, \mu_{j}, \ldots\right)_{j \in \mathbb{Z}_{1}^{d}}$. 
Lemma 4.8. Let $\Pi=[0,1]^{\mathrm{m}+\mathrm{n}}$. Then we have $\mathrm{X}_{\mathrm{P}} \in \mathcal{A}\left(\mathrm{l}^{\mathrm{a}}, \mathrm{l}^{\mathrm{a}}\right)$ and

$$
\left\|X_{P}\right\|_{D_{a}\left(s^{\prime}, r\right) \times \Pi} \leqslant C \varepsilon \quad\left\|\partial_{\zeta} X_{P}\right\|_{D_{a}\left(s^{\prime}, r\right) \times \Pi} \leqslant C \varepsilon .
$$

The proof of the above lemma is the same as one of lemma 3.1 and we omit it. Now we give the following lemma which will be applied in the sequel and will be proved in Appendix.

Lemma 4.9. Let $\gamma^{\prime}>0, \tau>m+n+1$, then for the parameter set $\Pi=[0,1]^{m+n}$, there is a subset $\Pi_{\gamma^{\prime}} \subset \Pi$ with

$$
\operatorname{meas}\left(\Pi \backslash \Pi_{\gamma^{\prime}}\right)=\mathrm{O}\left(\gamma^{\prime}\right),
$$

such that, for any $\zeta \in \Pi_{\gamma^{\prime}}$,

$$
|<k, \widehat{\omega}(\zeta)>+l| \geqslant \frac{\gamma^{\prime}}{(|k|+\delta(|k|))^{\tau-1}}, \quad \forall k \in \mathbb{Z}^{m+n}, l \in \mathbb{Z},|k|+|l| \neq 0,
$$

where $\delta(x)=1$ as $x=0$ and $\delta(x)=0$ as $x \neq 0$.

\section{Proof of main theorem}

In the following, we will verify Assumptions 4.1-4.6 for the above Hamiltonian (4.1).

Verifying Assumption 4.1: In view of (4.2), we have

$$
\frac{\partial \widehat{\omega}}{\partial \zeta}=\left(\begin{array}{cc}
\varepsilon I_{m} & 0 \\
\varepsilon \cdot \frac{\partial \breve{\omega}}{\partial \omega} & I_{n}
\end{array}\right), \text { for } \zeta \in \Pi,
$$

where $I_{m}$ denotes the unit $m \times m$-matrix. It is easy to check that $\operatorname{det}\left(\frac{\partial \widehat{\omega}}{\partial \zeta}\right) \neq 0$. Thus assumption 4.1 is verified.

Verifying Assumption 4.2: Take $\mathrm{L}=\mathrm{C} \varepsilon$, the proof is obvious.

Verifying Assumption 4.3: By Lemma 4.9, there exists a subset $\Pi_{\gamma^{\prime}} \subset \Pi$ with meas $\left(\Pi \backslash \Pi_{\gamma^{\prime}}\right)=\mathrm{O}\left(\gamma^{\prime}\right)$ such that for any $\zeta \in \Pi_{\gamma^{\prime}}$,

$$
|<k, \widehat{\omega}(\zeta)>+l+O(\varepsilon)| \geqslant \frac{\gamma^{\prime}}{(|k|+\delta(|k|))^{\tau}}, \quad \forall k \in \mathbb{Z}^{m+n}, l \in \mathbb{Z},|k|+|l| \neq 0,
$$

where $\varepsilon$ be small enough. Hence for any $\zeta \in \Pi_{\gamma^{\prime}}$,

$$
\begin{aligned}
&|<k, \widehat{\omega}(\zeta)>| \geqslant \frac{\gamma^{\prime}}{|k|^{\tau}}, \quad k \neq 0, \\
&\left|<k, \widehat{\omega}(\zeta)>+\widehat{\Omega}_{j}\right| \geqslant \frac{\gamma^{\prime}}{(|k|+\delta(|k|))^{\tau}}, \\
&\left|<k, \widehat{\omega}(\zeta)>+\widehat{\Omega}_{j}+\widehat{\Omega}_{l}\right| \geqslant \frac{\gamma^{\prime}}{(|k|+\delta(|k|))^{\tau}}, \\
&\left|<k, \widehat{\omega}(\zeta)>+\widehat{\Omega}_{j}-\widehat{\Omega}_{l}\right| \geqslant \frac{\gamma^{\prime}}{(|k|+\delta(|k|))^{\tau}}, \quad|k|+|| j|-| l|| \neq 0 .
\end{aligned}
$$

Assumption 4.3 is verified.

Verifying Assumption 4.4: Assumption 4.4 can be verified easily fulfilled by Lemma 4.8.

Verifying Assumption 4.5: From (2.53), we have

$$
\tilde{G}=\sum_{\alpha, \beta,|\alpha|+|\beta| \geqslant 4, \sum_{j \in \mathbb{Z}^{d}}\left(\alpha_{j}-\beta_{j}\right) j=0} \tilde{G}_{\alpha \beta}(\vartheta ; \omega) q^{\alpha} \bar{q}^{\beta}
$$


where

$$
\tilde{\mathrm{G}}_{\alpha \beta}=0 \quad \text { if } \sum_{j \in \mathbb{Z}^{\mathrm{d}}}\left(\alpha_{j}-\beta_{j}\right) j \neq 0 .
$$

Denote by $e_{s}$ the infinite dimensional vector with the $s^{\text {th }}$ component being 1 and the other components being zero, and $k_{2} \equiv\left(k_{21}, \ldots, k_{2 n}\right), k_{2 s}=\alpha_{i_{s}}-\beta_{i_{s}}, 1 \leqslant s \leqslant n, \bar{\alpha} \equiv\left(\ldots, \bar{\alpha}_{j}, \ldots\right)_{j \in \mathbb{Z}_{1}^{d}}, \bar{\beta} \equiv\left(\ldots, \bar{\beta}_{j}, \ldots\right)_{j \in \mathbb{Z}_{1}^{d}}$, $\bar{\alpha}_{j}=\alpha_{j}$ and $\bar{\beta}_{j}=\beta_{j}, j \in \mathbb{Z}_{1}^{\mathrm{d}}$ then

$$
\begin{aligned}
& \tilde{G}=\sum_{\sum_{j \in \mathbb{Z}^{d}}\left(\alpha_{j}-\beta_{j}\right) j=0} \varepsilon \tilde{G}_{\alpha \beta} q^{\alpha} \bar{q}^{\beta} \\
& =\sum_{\sum_{s=1}^{n}\left(\alpha_{i_{s}}-\beta_{i_{s}}\right) i_{s}+\sum_{j \in \mathbb{Z}_{1}^{\mathrm{d}}}\left(\alpha_{j}-\beta_{j}\right) j=0} \varepsilon \tilde{G}_{\alpha \beta} q_{i_{1}}^{\alpha_{i_{1}}} \bar{q}_{i_{1}}^{\beta_{i_{1}}} \cdots q_{i_{n}}^{\alpha_{i_{n}}} \bar{q}_{i_{n}}^{\beta_{i_{n}}} q^{\alpha-\sum_{s=1}^{n} \alpha_{i_{s}} e_{i_{s}}} \bar{q}^{\beta-\sum_{s=1}^{n} \beta_{i_{s}} e_{i_{s}}}
\end{aligned}
$$

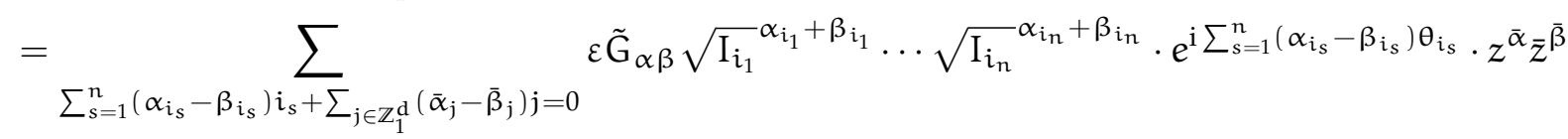

$$
\begin{aligned}
& :=\sum_{\sum_{s=1}^{n} k_{2 s} i_{s}+\sum_{j \in Z_{1}^{d}}\left(\bar{\alpha}_{j}-\bar{\beta}_{j}\right) j=0} P_{k_{2} b_{2} \bar{\alpha} \bar{\beta}}(\vartheta, J ; \zeta) I^{b_{2}} e^{i<k_{2}, \theta>} z^{\bar{\alpha}} \bar{z}^{\bar{\beta}} \equiv P .
\end{aligned}
$$

Thus

$$
P_{k_{2} b_{2} \bar{\alpha} \bar{\beta}}(\vartheta, J ; \zeta)=\varepsilon \tilde{G}_{\alpha \beta}, \quad P_{k_{2} b_{2} \bar{\alpha} \bar{\beta}}(\vartheta, J j \zeta)=0, \quad \text { if } \sum_{s=1}^{n} k_{2 s} i_{s}+\sum_{j \in \mathbb{Z}_{1}^{d}}\left(\bar{\alpha}_{j}-\bar{\beta}_{j}\right) j \neq 0,
$$

i.e., $\mathrm{P} \in \mathcal{D}$.

Verifying Assumption 4.6: By Lemma 2.1 (ii), we have for any fixed $j \in \mathbb{Z}_{1}^{\mathrm{d}}$ and $\tilde{\mathrm{c}} \in \mathbb{Z}^{\mathrm{d}} \backslash\{0\}$, the limits $\lim _{\tilde{\mathfrak{t}} \rightarrow \infty} \widetilde{\Omega}_{\mathfrak{j}+\tilde{\mathbf{c}} \tilde{\mathfrak{t}}}$ and $\lim _{\tilde{\mathfrak{t}} \rightarrow \infty} \partial_{\zeta} \widetilde{\Omega}_{\mathfrak{j}+\tilde{\mathbf{c}} \tilde{\mathfrak{t}}}$ exist and

$$
\left|\widetilde{\Omega}_{j+\tilde{\mathbf{c}} \tilde{\mathfrak{t}}}-\lim _{\tilde{\mathfrak{t}} \rightarrow \infty} \widetilde{\Omega}_{\mathfrak{j}+\tilde{\mathbf{c}} \tilde{\mathfrak{t}}}\right|_{\Pi}=\varepsilon^{(1+\rho)}\left\|\mu_{\mathfrak{j}+\tilde{\mathbf{c}} \tilde{\mathrm{t}}}^{*}-\lim _{\tilde{\mathfrak{t}} \rightarrow \infty} \mu_{\mathfrak{j}+\tilde{\mathbf{c}} \tilde{\mathrm{t}}}^{*}\right\|_{\Omega}^{*} \leqslant \frac{\varepsilon^{(1+\rho)}}{|\tilde{\mathfrak{t}}|} .
$$

By $P$ is just $\varepsilon \tilde{G}$ with the $\left(\vartheta, J, q_{i_{1}}, \cdots, q_{i_{n}}, \bar{q}_{i_{1}}, \cdots, \bar{q}_{i_{n}}, q_{j}, \bar{q}_{j}\right)$-variables expressed in terms of the $(\vartheta, J, \theta, I$, $z_{j}, \bar{z}_{j}$ )-variables and (2.55), we have for any fixed $j, l \in \mathbb{Z}_{1}^{\mathrm{d}}$ and $\tilde{\mathbf{c}} \in \mathbb{Z}^{\mathrm{d}} \backslash\{0\}$, the $\operatorname{limits} \lim _{\tilde{\mathfrak{t}} \rightarrow \infty} \frac{\partial^{2} \mathrm{P}}{\partial z_{j+\tilde{c} \mathfrak{t}} \partial z_{l-\tilde{c} \tilde{\mathfrak{t}}}}$ and $\lim _{\tilde{\mathfrak{t}} \rightarrow \infty} \frac{\partial^{2} \partial_{\zeta} P}{\partial z_{\mathfrak{j}+\tilde{\mathfrak{c}} \mathfrak{t}} \partial z_{l-\tilde{c} \tilde{\mathfrak{t}}}}$ exist and

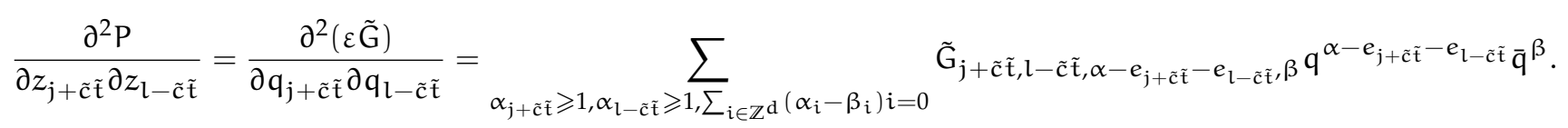

Thus,

$$
\begin{aligned}
& \left\|\frac{\partial^{2} P}{\partial z_{j+\tilde{\mathfrak{c}} \tilde{\mathfrak{t}}} \partial z_{l-\tilde{\mathbf{c}} \tilde{\mathfrak{t}}}}-\lim _{\tilde{\mathfrak{t}} \rightarrow \infty} \frac{\partial^{2} \mathrm{P}}{\partial z_{\mathfrak{j}+\tilde{\mathbf{c}} \tilde{\mathfrak{t}}} \partial \bar{z}_{l-\tilde{\mathbf{c}} \tilde{\mathfrak{t}}}}\right\|_{D_{\mathbf{a}}\left(s^{\prime}, \mathfrak{r}\right), \Pi}
\end{aligned}
$$

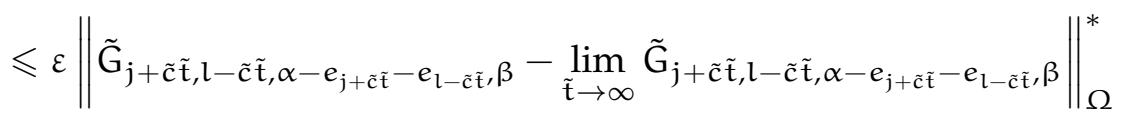

$$
\begin{aligned}
& \cdot\|q\|_{a} \cdot e^{-\left|\left(\sum_{i \in \mathbb{Z}^{d}}\left(\alpha_{i}-\beta_{i}\right) i\right)-(j+\tilde{c} \tilde{\mathfrak{t}}+l-\tilde{c} \tilde{\mathfrak{t}})\right| a} \leqslant \frac{\varepsilon^{(1+\rho)}}{|\tilde{\mathfrak{t}}|} e^{-|j+l| a} .
\end{aligned}
$$

Similarly, we have

$$
\left\|\frac{\partial^{2} P}{\partial z_{j}+\tilde{\mathfrak{c}} \tilde{\mathfrak{t}} \partial \bar{z}_{l+\tilde{\mathfrak{c}} \tilde{\mathfrak{t}}}}-\lim _{\tilde{\mathfrak{t}} \rightarrow \infty} \frac{\partial^{2} P}{\partial z_{j+\tilde{\mathfrak{c}} \tilde{\mathfrak{t}}} \partial \bar{z}_{l+\tilde{c} \tilde{\mathfrak{t}}}}\right\|_{D_{a}\left(s^{\prime}, r\right), \Pi} \leqslant \frac{\varepsilon^{(1+\rho)}}{|\tilde{\mathfrak{t}}|} e^{-|j-l| a},
$$


and

$$
\left\|\frac{\partial^{2} P}{\partial \bar{z}_{j+\tilde{c} \tilde{\mathfrak{t}}} \partial \bar{z}_{l-\tilde{c} \tilde{\mathfrak{t}}}}-\lim _{\tilde{\mathfrak{t}} \rightarrow \infty} \frac{\partial^{2} P}{\partial \bar{z}_{j+\tilde{\mathbf{c}} \tilde{\mathfrak{t}}} \partial \bar{z}_{l-\tilde{c} \tilde{\mathfrak{t}}}}\right\|_{D_{a}\left(s^{\prime}, r\right), \Pi} \leqslant \frac{\varepsilon^{(1+\rho)}}{|\tilde{\mathfrak{t}}|} e^{-|j+l| a} .
$$

Thus H satisfies Assumption 4.6.

By applying Theorem 4.7 ([17, Theorem 2]), we get Theorem 1.1.

\section{Appendix}

Proof of Lemma 4.9. Let

$$
\mathcal{R}_{k, l}^{2}=\left\{\zeta \in[0,1]^{m+n}:|<k, \widehat{\omega}(\zeta)>+l|<\frac{\gamma^{\prime}}{(|k|+\delta(|k|))^{\tau-1}}\right\}
$$

and

$$
\bar{\Pi}=\bigcup_{k \in \mathbb{Z}^{m+n}} \bigcup_{l \in \mathbb{Z},|k|+|l| \neq 0} \mathcal{R}_{k, l}^{2} .
$$

When $k \neq 0$, by the Fubini theorem to estimate $\operatorname{meas} \mathcal{R}_{k, l}^{2}$ it is sufficient to estimate the one-dimensional measure of the intersection of $\mathcal{R}_{k, l}^{2}$ with every line parallel to some fixed direction. In particular, to the direction given by the vector $k|k|^{-1}$. The intersection of $\mathcal{R}_{k, l}^{2}$ with the line $L_{\tilde{\eta}}=\left\{\tilde{\eta}+t k|k|^{-1}: t \in \mathbb{R}\right\}, \tilde{\eta} \in$ $\mathbb{R}^{\mathrm{m}+\mathrm{n}}$, is equal to the set

$$
\left\{t \in \mathbb{R}:|\mathcal{T}(\mathrm{t})|<\frac{\gamma^{\prime}}{(|k|+\delta(|k|))^{\tau-1}}\right\}
$$

where

$$
\mathcal{T}(\mathrm{t})=\left.(<\mathrm{k}, \widehat{\omega}(\zeta)>+l)\right|_{\omega=\tilde{\eta}+\mathrm{tk}|\mathrm{k}|^{-1}}
$$

Observe that $(\partial / \partial t) \omega \cdot k=|k|$, so for $t_{1}>t_{2}$ we have

$$
\mathcal{T}\left(t_{1}\right)-\mathcal{T}\left(t_{2}\right)=<k,\left(t_{1}-t_{2}\right) k|k|^{-1}>=|k|\left(t_{1}-t_{2}\right) .
$$

Thus, the measure of the set (5.1) is no larger than $\frac{\gamma^{\prime}|k|^{-1}}{(|k|+\delta(|k|))^{\tau-1}}$ by Appendix $C$ in [18]. This estimate jointly with the Fubini theorem implies that

$$
\operatorname{meas} \mathcal{R}_{\mathrm{k}, \mathrm{l}}^{2} \leqslant \frac{\gamma^{\prime}|\mathrm{k}|^{-1}}{(|\mathrm{k}|+\delta(|\mathrm{k}|))^{\tau-1}}
$$

Let $k=0$, then

$$
|<k, \widehat{\omega}(\zeta)>+l|=|l| \geqslant 1>\gamma^{\prime}
$$

When $|l|>2+|k||\widehat{\omega}|$, we have

$$
|<k, \widehat{\omega}(\zeta)>+l| \geqslant|l|-(1+|k||\widehat{\omega}|)>1,
$$

which implies the sets $\mathcal{R}_{k, l}^{2}$ are empty. Set

$$
|k|_{\infty}=\max \left\{\left|k_{1}\right|,\left|k_{2}\right|, \ldots,\left|k_{m+n}\right|\right\},
$$

note that

$$
\sum_{|k|_{\infty}=p} 1 \leqslant 2(m+n)(2 p+1)^{m+n-1,}|k|_{\infty} \leqslant|k| \leqslant(m+n)|k|_{\infty},
$$


we obtain

$$
\begin{aligned}
\operatorname{meas} \bar{\Pi} & =\operatorname{meas} \bigcup_{0 \neq k \in \mathbb{Z}^{\mathrm{m}+\mathrm{n}}} \bigcup_{l=-2-[[|k||\widehat{\omega}|]]}^{2+[[|k| \widehat{\omega} \mid]]} \mathcal{R}_{k, l}^{2} \\
& \leqslant \sum_{0 \neq k \in \mathbb{Z}^{\mathrm{m}+\mathrm{n}}}(4+2[[|k||\widehat{\omega}|]]) \frac{\gamma^{\prime}|\mathrm{k}|^{-1}}{(|k|+\delta(|k|))^{\tau-1}} \\
& \leqslant \mathrm{C}_{0} \sum_{0 \neq k \in \mathbb{Z}^{\mathrm{m}+\mathrm{n}}} \frac{\gamma^{\prime}}{|k|^{\tau-1}} \\
& \leqslant \mathrm{C}_{0}(\mathrm{~m}+\mathrm{n}) \gamma^{\prime} \sum_{p=1}^{\infty}(2 p+1)^{m+n-1} p^{-(\tau-1)},
\end{aligned}
$$

where $[[\bullet]]$ stands for the integer part of $\bullet$, and $C_{0}$ is a constant depending on $|\widehat{\omega}|$ only. Because series $\sum_{p=1}^{\infty}(2 p+1)^{m+n-1} p^{-(\tau-1)}$ is convergent for $\tau>m+n+1$, we have meas $\bar{\Pi}=\mathrm{O}\left(\gamma^{\prime}\right)$. Let $\Pi_{\gamma^{\prime}}=\Pi \backslash \bar{\Pi}$ we have (4.4) and (4.5). This completes the proof.

\section{Acknowledgment}

This paper is partially supported by the Natural Science Foundation of Shandong Province, China (Grant No.ZR2016AQ25) and the National Natural Science Foundation of China (Grant No.11501571).

\section{References}

[1] D. Bambusi, S. Graffi, Time quasi-periodic unbounded perturbations of Schrödinger operators and KAM methods, Comm. Math. Phys., 219 (2001), 465-480. 1, 1

[2] M. Berti, P. Bolle, Sobolev quasi-periodic solutions of multidimensional wave equations with a multiplicative potential, Nonlinearity, 25 (2012), 2579-2613. 1

[3] M. Berti, P. Bolle, Quasi-periodic solutions with Sobolev regularity of NLS on $\mathbb{T}^{\mathrm{d}}$ with a multiplicative potential, J. Eur. Math. Soc. (JEMS), 15 (2013), 229-286. 1, 1

[4] J. Bourgain, Construction of quasi-periodic solutions for Hamiltonian perturbations of linear equations and applications to nonlinear PDE, Internat. Math. Res. Notices, 1994 (1994), 21 pages. 1

[5] J. Bourgain, Construction of periodic solutions of nonlinear wave equations in higher dimension, Geom. Funct. Anal., 5 (1995), 629-639.

[6] J. Bourgain, Quasi-periodic solutions of Hamiltonian perturbations of 2D linear Schrödinger equations, Ann. of Math., 148 (1998), 363-439. 1

[7] J. Bourgain, Nonlinear Schrödinger equations, Hyperbolic equations and frequency interactions, Park City, UT, (1995), IAS/Park City Math. Ser., Amer. Math. Soc., Providence, RI, 5 (1999), 3-157.

[8] J. Bourgain, Green's function estimates for lattice Schrödinger operators and applications, Annals of Mathematics Studies, Princeton University Press, Princeton, NJ, (2005). 1

[9] W. Craig, C. E. Wayne, Newton's method and periodic solutions of nonlinear wave equations, Comm. Pure Appl. Math., 46 (1993), 1409-1498. 1

[10] H. L. Eliasson, S. B. Kuksin, On reducibility of Schrödinger equations with quasiperiodic in time potentials, Comm. Math. Phys., 286 (2009), 125-135. 1

[11] H. L. Eliasson, S. B. Kuksin, KAM for the nonlinear Schrödinger equation, Ann. of Math., 172 (2010), 371-435. 1

[12] J.-S. Geng, X.-D. Xu, J.-G. You, An infinite dimensional KAM theorem and its application to the two dimensional cubic Schrödinger equation, Adv. Math., 226 (2011), 5361-5402. 1

[13] J.-S. Geng, Y.-F. Yi, Quasi-periodic solutions in a nonlinear Schrödinger equation, J. Differential Equations, 233 (2007), 512-542. 1

[14] J.-S. Geng, J.-G. You, A KAM theorem for one dimensional Schrödinger equation with periodic boundary conditions, J. Differential Equations, 209 (2005), 1-56. 1

[15] J.-S. Geng, J.-G. You, A KAM theorem for Hamiltonian partial differential equations in higher dimensional spaces, Comm. Math. Phys., 262 (2006), 343-372. 1

[16] J.-S. Geng, J.-G. You, KAM tori for higher dimensional beam equations with constant potentials, Nonlinearity, 19 (2006), 2405-2423. 1 
[17] J.-S. Geng, J.-G. You, KAM theorem for higher dimensional nonlinear Schrödinger equations, J. Dynam. Differential Equations, 25 (2013), 451-476. 1, 1, 1, 3, 4, 4, 4.7, 5

[18] S. B. Kuksin, Nearly integrable infinite-dimensional Hamiltonian systems, Lecture Notes in Mathematics, SpringerVerlag, Berlin, (1993). 1, 2, 5

[19] S. B. Kuksin, J. Pöschel, Invariant Cantor manifolds of quasi-periodic oscillations for a nonlinear Schrödinger equation, Ann. of Math., 143 (1996), 149-179. 1

[20] Z.-G. Liang, J.-G. You, Quasi-periodic solutions for 1D Schrödinger equations with higher order nonlinearity, SIAM J. Math. Anal., 36 (2005), 1965-1990.

[21] J. Pöschel, A KAM-theorem for some nonlinear partial differential equations, Ann. Scuola Norm. Sup. Pisa Cl. Sci., 23 (1996), 119-148. 1

[22] J. Pöschel, Quasi-periodic solutions for a nonlinear wave equation, Comment. Math. Helv., 71 (1996), 269-296. 1, 2

[23] C. Procesi, M. Procesi, A KAM algorithm for the resonant non-linear Schrödinger equation, Adv. Math., 272 (2015), 399-470. 1

[24] C. E. Wayne, Periodic and quasi-periodic solutions of nonlinear wave equations via KAM theory, Comm. Math. Phys., 127 (1990), 479-528. 1

[25] J.-X. Xu, J.-G. You, Persistence of lower-dimensional tori under the first Melnikov's non-resonance condition, J. Math. Pures Appl., 80 (2001), 1045-1067. 1

[26] X.-P. Yuan, Quasi-periodic solutions of completely resonant nonlinear wave equations, J. Differential Equations, 230 (2006), 213-274. 1, 2 